This article has been accepted for publication in Monthly Notices of the Royal Astronomical Society (C: 2019 The Authors Published by Oxford University Press on behalf of the Royal Astronomical Society. All rights reserved. 


\title{
Polarization of radio relics in galaxy clusters
}

\author{
D. Wittor, ${ }^{1,2,3 \star}$ M. Hoeft, ${ }^{4}$ F. Vazza ${ }^{\oplus,},{ }^{1,2,3}$ M. Brüggen ${ }^{3}$ and P. Domínguez-Fernández ${ }^{3}$ \\ ${ }^{1}$ Dipartimento di Fisica e Astronomia, Universita di Bologna, Via Gobetti 93/2, 40122, Bologna, Italy \\ ${ }^{2}$ INAF, Istituto di Radioastronomia di Bologna, via Gobetti 101, I-41029 Bologna, Italy \\ ${ }^{3}$ Hamburger Sternwarte, Gojenbergsweg 112, 21029 Hamburg, Germany \\ ${ }^{4}$ Thüringer Landessternwarte, Sternwarte 5, 07778 Tautenburg, Germany
}

Accepted 2019 September 23. Received 2019 September 18; in original form 2019 January 14

\begin{abstract}
Radio emission in the form of giant radio relics is observed at the periphery of galaxy clusters. This non-thermal emission is an important tracer for cosmic ray electrons and intracluster magnetic fields. One striking observational feature of these objects is their high degree of polarization, which provides information on the magnetic fields at the relics' positions. In this contribution, we test if state-of-the-art high resolution cosmological simulations are able to reproduce the polarization features of radio relics. Therefore, we present a new analysis of high-resolution cosmological simulations to study the polarization properties of radio relics in detail. In order to compare our results with current and future radio observations, we create mock radio observations of the diffuse polarized emission from a massive galaxy cluster using six different projections, for different observing frequencies and for different telescopes. Our simulations suggest that, due to the effect of Faraday rotation, it is extremely difficult to relate the morphology of the polarized emission for observing frequencies below $1.4 \mathrm{GHz}$ to the real magnetic field structure in relics. We can reproduce the observed degree of polarization and also several small-scale structures observed in real radio relics, but further work would be needed to reproduce some large-scale spectacular features as observed in real radio relics, such as the 'Sausage' and 'Toothbrush' relics.
\end{abstract}

Key words: MHD-polarization-galaxies: clusters-galaxies: clusters: individual: radio relics.

\section{INTRODUCTION}

Radio observations detect diffuse radio emission in the form of radio relics and radio haloes in galaxy clusters (e.g. Ferrari et al. 2008; van Weeren et al. 2019). Radio haloes fill the clusters' central regions and they are believed to be connected to turbulence in the intracluster medium (ICM). Radio relics are found at the cluster periphery co-located with shock waves observed in X-rays (e.g. Markevitch et al. 2005; Botteon et al. 2016). Hence, it is assumed that shock (re-)acceleration of cosmic ray electrons produces radio relics (e.g. Ensslin et al. 1998; Hoeft \& Brüggen 2007; Brunetti \& Jones 2014; Nuza et al. 2017, and references therein). It is widely accepted that diffusive shock acceleration (DSA) can accelerate the observed radio-emitting electrons (e.g. Blandford \& Ostriker 1978), yet the lack of detected $\gamma$-ray emission, a by-product of proton acceleration (e.g. Ackermann et al. 2014), questions the viability of the DSA model for typical cluster shocks (e.g. Vazza \& Brüggen 2014; Vazza et al. 2015). This triggered the exploration of new scenarios in which the role of the magnetic field topology at shock fronts can control the efficiencies of electron and proton acceleration (e.g. Caprioli \& Spitkovsky 2014b; Guo, Sironi \& Narayan 2014, and references therein), as we tested in detail in Wittor, Vazza \& Brüggen (2017). More recently, Botteon et al. (2019) have found evidence that an efficiency $\geq 100$ per cent is required to explain the observed radio power of relics with low Mach number (i.e. $\leq 2$ ).

One striking observational feature of radio relics is their high degree of polarization, which is in contrast to the almost complete absence of polarized emission in radio haloes (e.g. Govoni et al. 2013, and references therein). The mechanism producing this high degree of polarization is still debated: either it is caused by regular large-scale magnetic fields or by the compression of tangled smallscale magnetic fields (Laing 1980). The polarization of radio relics has been studied by a number of authors ${ }^{1}$ (e.g. Bonafede et al. 2009b; van Weeren et al. 2010, 2012; Kale et al. 2012; de Gasperin et al. 2015). These observations have shown that the average degree of polarization ranges from a few up to $\sim 60$ per cent. In some radio relics, the $B$-vector of the polarized synchrotron emission

\footnotetext{
${ }^{1}$ Table 1 provides an overview of known relics that have been studied in polarization. We devote Section 1.1 to describing these in more detail.
} 
is perpendicular to the shock normal. However, some radio relics show a more complex polarization structure. If the high degree of polarization is caused by a regular large-scale magnetic field, then the alignment does not depend on the shock morphology. On the other hand, if the compression of small-scale magnetic fields is responsible for the high degree of polarization, the magnetic field should always be perpendicular to the shock normal. A simple model (e.g. Ensslin et al. 1998) shows that a moderately strong shock may explain the degree of polarization (see fig. 12 in Kierdorf et al. 2017). In a more elaborate scenario, Iapichino \& Brüggen (2012) showed that magnetic amplification due to compression at shocks can explain the high polarization in radio relics (see also Ensslin et al. 1998), even though this was based on analytical arguments only and neglected the effect of the viewing angle. With increasing resolution and sensitivity, radio telescopes (e.g. Square Kilometre Array and Very Large Array) will be able to study the polarization of radio sources with unprecedented detail (e.g. Johnston-Hollitt et al. 2015)

To our knowledge, only Skillman et al. (2013) have studied the polarization of radio relics in cosmological magnetohydrodynamical simulations at an observing frequency of $v_{\mathrm{obs}}=$ 1.4 GHz. In their model, they assign all the radio emission to the shock front using equation (31) of Hoeft \& Brüggen (2007) and they estimate the corresponding polarized emission using the notation of Otmianowska-Mazur et al. (2009). Yet, ageing of cosmic ray electrons and corresponding downstream effects on the radio emission are neglected. Their results show a significant variation of the polarization, both across and along the relic with a peak polarization of approximately 75 per cent. Furthermore, they showed that the polarization direction is less coherent if the relic is seen face-on instead of edge-on.

In this contribution, we use ENZO cosmological magnetohydrodynamical simulations of a merging galaxy cluster to analyse the radio properties of one radio relic seen in different projections. We compute the downstream emission of this relic at different frequencies and model the polarized emission using the formalism derived by Burn (1966). This work is meant to be a pilot study to explore the degree of realism of magnetic fields in high-resolution cosmological simulations and to present our test suite.

This work is structured as follows: we complete this section by giving an overview of the available polarization observations of radio relics. In Section 2.1, we describe our simulation and analysis tools. In Sections 2.2 and 2.3, we describe the models we use to compute the polarized emission. The results are presented in Section 3. Sections 3.1, 3.2 and 3.3 give a detailed description of our simulated radio relic, its local magnetic field and its rotation measures, respectively. The results on the polarized emission are presented in Section 3.4. We present our mock observations of the radio relic in Section 3.5. In Section 3.6, we highlight morphological features of our radio relic, as found in highly resolved observations. In Section 3.7, we discuss how well the polarized emission represents the magnetic field structure at the relic. We summarize and conclude our work in Section 4.

\subsection{Polarization properties of observed radio relics}

While the statistical properties of the continuum emission from radio relics are relatively well known (e.g. Nuza et al. 2017; Golovich et al. 2019), far less is known about the properties of the polarized emission. Currently, there are only 20 galaxy clusters hosting radio relics (half of which are double radio relics, e.g. Bonafede et al. 2009b; Lindner et al. 2014) that have been observed in polarization. In Table 1, we summarize the main properties of these observations. These relics have been detected at redshifts ranging from $z \approx 0.04$ to $z \approx 0.55$ and their largest linear sizes (LLS) lie in the range of a few hundred kpc to a few Mpc. Most of these relics are found at distances of $0.5-2 \mathrm{Mpc}$ from the cluster centre. We have not found Mach number estimates for all relics, but the available Mach numbers are all in the range of $M \approx 1.7-4.6$.

The average degree of polarization varies between a few per cent up to $\sim 60$ per cent, as in the case of CIZA J2242.8+5301 (see van Weeren et al. 2010). Locally, the observed degree of polarization can be as high as $\sim 70$ per cent as in Abell $1240^{2}$ (see Bonafede et al. 2009b).

The relic population shows a vast range of orientation of the polarization vectors. In the cluster CIZA J2242.8+5301, the polarization $E$-vectors align perfectly with the shock normal across the entire length of the relic, $\sim 2 \mathrm{Mpc}$ (see van Weeren et al. 2010), and other relics, such as Abell 2744 (see Pearce et al. 2017) or $\mathrm{ZwCl}$ 0008.8+5215 (see van Weeren et al. 2011b; Golovich et al. 2017) show similar behaviour. For a sufficiently strong shock, the magnetic field compression at the shock front could explain the alignment of the shock normal and the polarization $E$-vector. On the other hand, such alignment is more difficult to achieve for a large-scale magnetic field, as will be shown in this work.

The morphology of the polarized emission in other relics is more complex. The orientation of the polarization vectors of radio relics, such as MACS J0717+3745 (see Bonafede et al. 2009a), changes rapidly over the length of the relic. Therefore, their polarized structures are not uniform and they appear rather patchy. At the same time, there is no obvious alignment between the polarization vector and the shock normal, making it unclear if the shock is simply too weak to align the magnetic field with the shock normal.

In other cases, such as Abell 3744, PSZ1 G096.89+24.17 or PSZ1 G108.18-11.53 (see Kale et al. 2012; de Gasperin et al. 2014, 2015, respectively), the polarized emission is not detected across the whole relic and the morphology seems to be patchy as well. Thus, it is uncertain if the polarization is uniform across the relic.

Finally, it is difficult to determine the shock direction in relics like Abell 548b or Abell 2256 (see Feretti et al. 2006; Clarke \& Ensslin 2006, respectively), and hence one cannot correlate the polarization vectors with the shock direction. The shock morphology might directly affect the polarization morphology. A spherical-cap-type shock would produce aligned polarization vectors, while a distorted shock structure could cause patchy polarized emission.

In summary, the polarized emission of radio relics shows a wide range of properties. It seems that the observed degree of polarization does not solely depend on one parameter, such as the telescope configuration (i.e. observing frequency and beam size) or the physical properties of the relic (i.e. distance to the cluster centre, Mach number, LLS or redshift). Clearly, the degree of polarization increases with frequency (smaller wavelengths) as Faraday rotation, $\propto \mathrm{RM} \lambda^{2}$, becomes non-relevant. This was shown by Shimwell et al. (2015) who observed the Bullet cluster at different frequencies but keeping a constant beam size. On the other hand, beam depolarization can reduce the degree of polarization even at high frequencies. Several relics, such as CIZA J2242.8+5301, show

\footnotetext{
${ }^{2}$ However, the uncertainty of these measurements is fairly large, as Abell 1240 is very faint compared to other relics. Bonafede et al. (2009b) measured fluxes of $\sim 6 \mathrm{mJy}$ and $\sim 10 \mathrm{mJy}$ for the two relics, while Loi et al. (2017) measured a flux of $\sim 161 \mathrm{mJy}$ for the relic in CIZA J2242.8+5301.
} 
Table 1. The table shows various properties of observed relics that have been studied in polarization. The columns provide: the relic name, the redshift, the estimated Mach number, observing frequency and beam size, followed by the average and the maximum polarization fraction of the relic. We also give the largest linear size (LLS) and the distance to the cluster centre $\left(d_{\mathrm{c}}\right)$ as found in the literature. The table is complemented by references. We did not find both average and maximum degree of polarization for all relics. The maximum values of the polarization are difficult to measure so they should be taken with caution. The references given in the last column refer to the measurements of the polarization fraction. All other quantities, if not stated otherwise, have been derived or quoted in these references. We put as an additional caveat that the Mach numbers might not have been computed in the same way. So, one should compare these values with caution as there is a bias in the Mach number estimations; see Section 3.1.

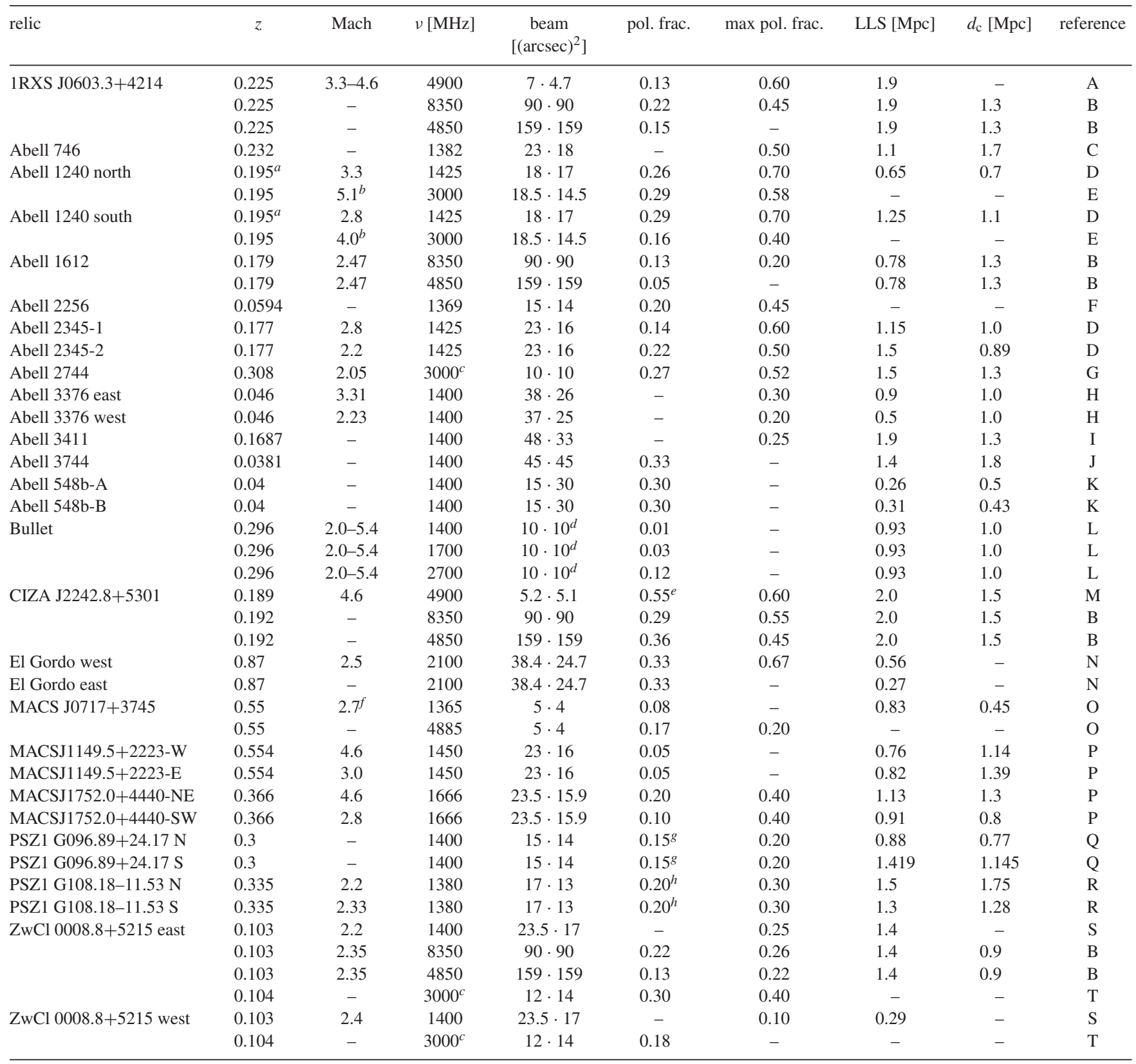

Notes. ${ }^{a}$ Taken from Golovich et al. (2018); ${ }^{b}$ we cite the Mach number derived from the integrated spectral index, while Hoang et al. (2018) also use the injection spectral index to compute the Mach number. Using the injection spectral index, they obtain Mach numbers of $\sim 2.4$ and $\sim 2.3$ for Abell 1240-1 and Abell 1240-2 respectively; ${ }^{c}$ both Golovich et al. (2017) and Pearce et al. (2017) only provide a frequency range, 2000-4000 GHz; hence, we assume the mean of $3000 \mathrm{GHz} ;{ }^{d}$ we assume this value, following the caption of fig. 3 in Shimwell et al. (2015); ${ }^{e}$ we give the arithmetic mean of the range, $0.5-0.6$, given in van Weeren et al. (2010); ${ }^{t}$ taken from van Weeren et al. (2017); ${ }^{g}$ we give the arithmetic mean of the range, $0.1-0.2$, given in de Gasperin et al. (2014); ${ }^{h}$ we give the arithmetic mean of the range, 0.1-0.3, given in de Gasperin et al. (2015).

References: A: van Weeren et al. (2012); B: Kierdorf et al. (2017); C: van Weeren et al. (2011a); D: Bonafede et al. (2009b); E: Hoang et al. (2018); F: Clarke \& Ensslin (2006); G: Pearce et al. (2017); H: Kale et al. (2012); I: van Weeren et al. (2013); J: Rudnick \& Brown (2009); K: Feretti et al. (2006); L: Shimwell et al. (2015); M: van Weeren et al. (2010); N: Lindner et al. (2014); O: Bonafede et al. (2009a); P: Bonafede et al. (2012); Q: de Gasperin et al. (2014); R: de Gasperin et al. (2015); S: van Weeren et al. (2011b); T: Golovich et al. (2017). 

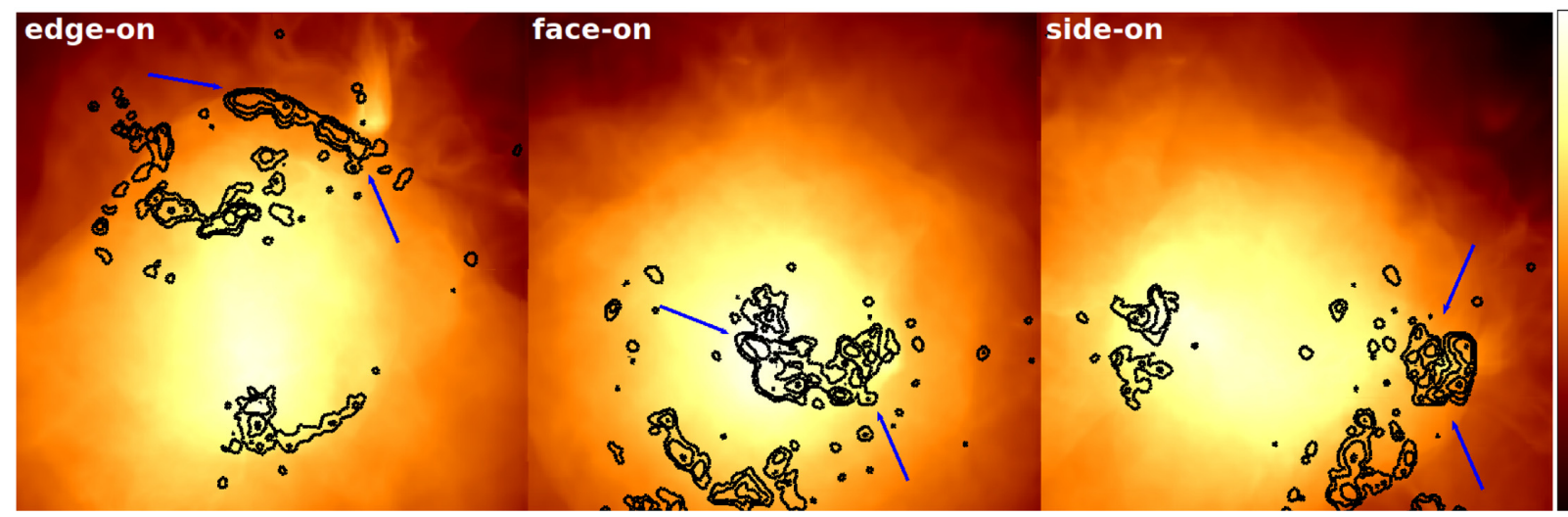

Figure 1. Projected baryonic density (colour) overlaid with the radio contours at $v_{\mathrm{obs}}=1.4 \mathrm{GHz}$. The density is given in units of $\log _{10}\left(\mathrm{~g} \mathrm{~cm}^{-3}\right)$. The radio contours are plotted at $4 \times\left[10^{24}, 10^{25}, 10^{26}, 10^{27}\right] \mathrm{erg} \mathrm{s}^{-1} \mathrm{~Hz}^{-1}$ per pixel. The different panels show the projections along the three different lines of sight. The solid blue arrows mark the position of the brightest radio relic as seen in the different projections. (A colour version is available in the online article.)

this effect. Van Weeren et al. (2010) observed this relic at $4.9 \mathrm{GHz}$ obtaining an average polarization fraction of 0.55 , while Kierdorf et al. (2017) obtained a lower degree of polarization at $4.85 \mathrm{GHz}$ and $8.35 \mathrm{GHz}$ but using larger beam sizes.

\section{SIMULATION SET-UP}

\subsection{ENZO}

In this work, we run cosmological simulations with the ENZO code (Bryan et al. 2014), which uses an $N$-body particle-mesh solver to simulate the dark matter component (Hockney \& Eastwood 1988) and an adaptive mesh method to follow the baryonic component (Berger \& Colella 1989). To solve for the magneto-hydrodynamical equations (see section 2.1 in Bryan et al. 2014), we use the piecewise linear method (Colella \& Glaz 1985) in combination with the hyperbolic Dedner cleaning (Dedner et al. 2002).

We focus on analysing one massive, $\sim 10^{15} \mathrm{M}_{\odot}$ (at $z=0$ ), galaxy cluster drawn from a large sample of simulations, as detailed in Vazza et al. (2018b) and Domínguez-Fernández et al. (2019). When analysed with our shock finder (see below) this cluster hosts a few shock waves in its periphery that could produce prominent radio relics, with typical large-scale morphologies of real radio relics (see Fig. 1).

Our simulation starts from a root grid with a comoving size of $\sim(260 \mathrm{Mpc})^{3}$ that is sampled with $256^{3}$ cells and $256^{3}$ dark matter particles. We further refined a comoving volume of approximately $(25 \mathrm{Mpc})^{3}$ centred around the galaxy cluster $2^{8}$ times, using eight levels of adaptive mesh refinement (AMR), for a final resolution of $\Delta x=3.95 \mathrm{kpc}$. For the analysis we used the $7.9 \mathrm{kpc}$ reconstruction of the grid, as the relic region is lying at the border of the highest AMR region.

The simulation ran from redshift $z=30$ to redshift $z=0$. At redshift $z=30$, we seeded a uniform primordial field with a comoving value of $B_{0}=0.1 \mathrm{nG}$. For the various cosmological parameters we chose: $H_{0}=72.0 \mathrm{~km} \mathrm{~s}^{-1} \mathrm{Mpc}^{-1}, \Omega_{\mathrm{M}}=0.258, \Omega_{\mathrm{b}}=$ $0.0441, \Omega_{\Lambda}=0.742$ and $\sigma_{8}=0.8$.

In order to find shock waves that are able to produce radio relics, we apply a velocity jump method following Vazza, Brunetti \& Gheller (2009). This approach measures the Mach numbers along the three coordinate axes of the simulation based on the threedimensional velocity information, and the final Mach number is computed as $M=\sqrt{M_{x}^{2}+M_{y}^{2}+M_{z}^{2}}$. For further analysis, we stored the three components of the Mach number, as they provide information on the propagation direction of the shock and of the shock normal in each shocked cell $\mathbf{n}_{\text {shock }}=\left(M_{x}, M_{y}, M_{z}\right)^{\top}$.

\subsection{Synchrotron emission}

In the following section, we give an overview of the model to compute the radio emission. For more details, we direct readers to Appendix A. We compute the downstream profile of the synchrotron emission from shocked cells following the approach of Hoeft \& Brüggen (2007).

For simplicity, we assume that the properties of the shock front do not change within the electron cooling time. Hence, both the magnetic field strength and the downstream temperature at the shock determine the downstream profile. This assumption is crude as both quantities might affect the shape of the profile. Yet in most cases, the downstream width at frequencies $v_{\text {obs }}>1.0 \mathrm{GHz}$ is smaller than both the physical resolution of the simulation and the effective resolution of the MHD scheme ${ }^{3}$ (see Fig. 2). Hence, constant shock properties are a valid assumption for frequencies above $1.0 \mathrm{GHz}$.

For each shocked cell, we compute the downstream profile of the radio emission as a function of the distance to the shock front. The emission per volume at a distance $x$ is the convolution of the electron spectrum $n_{\mathrm{E}}(\tau, x)$ and the modified Bessel function $F\left(1 / \tau^{2}\right)$ :

$\frac{\mathrm{d} P}{\mathrm{~d} V \mathrm{~d} \nu}(x)=C_{\mathrm{R}} \int_{0}^{E_{\max }} n_{\mathrm{E}}(\tau, x) F\left(\frac{1}{\tau^{2}}\right) \mathrm{d} \tau$.

The electron spectrum at a distance $x$ from the shock is thus:

$$
\begin{aligned}
n_{\mathrm{E}}(E, x)= & \frac{n_{\mathrm{e}} C_{\text {spec }}}{m_{\mathrm{e}} c^{2}}\left(\frac{E}{m_{\mathrm{e}} c^{2}}\right)^{-s} \\
& \times\left[1-\left(\frac{m_{\mathrm{e}} c^{2}}{E_{\max }}+C_{\text {cool }} \frac{x}{v_{\mathrm{d}}}\right) \frac{E}{m_{\mathrm{e}} c^{2}}\right]^{s-2} .
\end{aligned}
$$

In the equations above, $\tau$ depends on the electron energy. Electrons are considered to be suprathermal if their energy is above $E_{\min }=$ $10 k_{\mathrm{b}} T$, using the Boltzmann constant $k_{\mathrm{b}}$, and they are accelerated

\footnotetext{
${ }^{3}$ See Section 4 for a more detailed discussion on the resolution of the Dedner cleaning procedure, which is $\sim 32 \mathrm{kpc}$.
} 


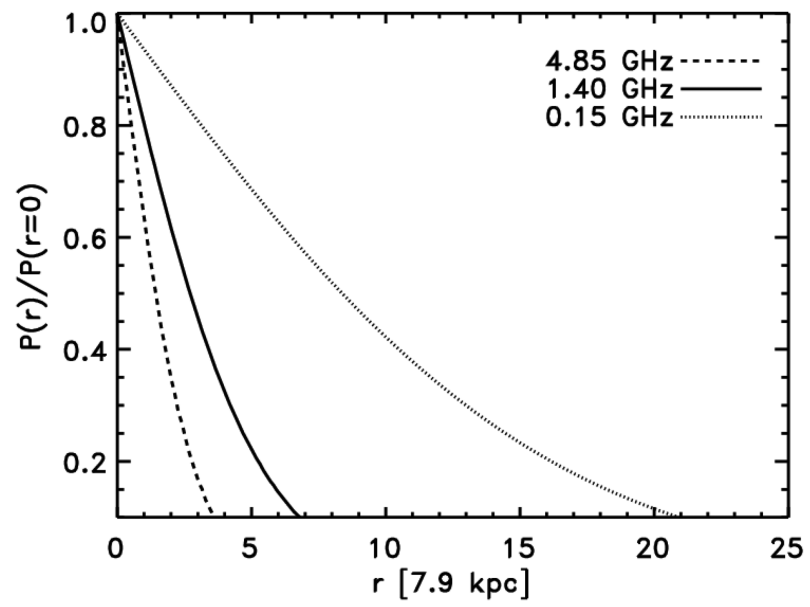

Figure 2. Example of the downstream profiles of the radio emission at $v=0.15 \mathrm{GHz}$ (dotted), $1.4 \mathrm{GHz}$ (solid) and $4.85 \mathrm{GHz}$ (dashed). The profiles have been normalized to the radio emission at the shock front. The shock properties are: $M=2.3, T_{\mathrm{d}}=1.2 \times 10^{8} \mathrm{~K}$ and $B=0.39 \mu \mathrm{G}$. The $x$-axis is in units of the grid resolution $\mathrm{d} x=7.9 \mathrm{kpc}$.

to a finite energy $E_{\max }$. Therefore, we only compute the spectrum if $E C_{\text {cool }} x / v_{\mathrm{d}} / m_{\mathrm{e}} / c^{2}<1-E / E_{\max }$, with the downstream velocity $v_{\mathrm{d}}$ and the cooling constant $C_{\text {cool }}$ (see Appendix A).

The normalization of the spectrum $C_{\text {spec }}$ depends on the acceleration efficiency $\xi_{\mathrm{e}}$. In the framework of DSA, it is difficult to reach the observed radio luminosities using weak Mach numbers to inject electrons from the thermal pool, as the resulting particle distributions are steep. In order to produce an observable radio relic, we assume an acceleration efficiency of $\xi_{\mathrm{e}}=0.02$. Furthermore, we include re-acceleration following the approach of Vazza et al. (2015). Hence, we assume that shock accelerates a distribution of pre-existing 'fossil' cosmic ray electrons (e.g. Pinzke, Oh \& Pfrommer 2013), which boosts the emission by a factor of $\approx 100$, for the considered Mach number regime, compared to the singleinjection case in Hoeft \& Brüggen (2007). In the downstream, we compute the radio emission on nodes that lie along the shock normal and that have a fixed distance of $\mathrm{d} x=1 \mathrm{kpc}$. We assign the emission to the grid cell that hosts the node, assuming that the shock surface matches the cell surface. The emission volume at each node is thus $V_{\mathrm{em}}=(7.9 \mathrm{kpc})^{2} \cdot 1 \mathrm{kpc} \approx 62.4 \mathrm{kpc}^{3}$. In Fig. 2 , we give an example of the downstream radio-emission profile produced by the same shock, $M=2.3$, at three different frequencies. As the width of the downstream profile changes with frequency, the total volume of the relic increases with decreasing frequency.

We use the same algorithm to compute the parallel, $P_{\perp}$, and perpendicular, $P_{\|}$, components of the radio emission as:

$\frac{\mathrm{d} P_{\perp}}{\mathrm{d} V \mathrm{~d} \nu}(x)=C_{\mathrm{R}} \int_{0}^{E_{\max }} n_{\mathrm{E}}(\tau, x)\left[F\left(\frac{1}{\tau^{2}}\right)-G\left(\frac{1}{\tau^{2}}\right)\right] \mathrm{d} \tau$
$\frac{\mathrm{d} P_{\|}}{\mathrm{d} V \mathrm{~d} \nu}(x)=C_{\mathrm{R}} \int_{0}^{E_{\max }} n_{\mathrm{E}}(\tau, x)\left[F\left(\frac{1}{\tau^{2}}\right)+G\left(\frac{1}{\tau^{2}}\right)\right] \mathrm{d} \tau$.

The functions $F(x)$ and $G(x)$ depend on the modified Bessel functions (see Appendix A).

After applying the above-described algorithm, multiple grid cells contain radio emission. For our analysis, we will only use cells that fulfil two conditions: the emissivity on the three-dimensional grid has to be larger than zero and the cell has to contribute to a bright pixel in the radio map with a luminosity above $4 \times 10^{24} \mathrm{erg} \mathrm{s}^{-1} \mathrm{~Hz}^{-1}$, i.e. the sum of the emission of all cells that contribute to the pixel has to be larger than that value. To describe the characteristic physical properties of the radio relic and to provide a sense of what would dominate observations, we introduce, in addition to the arithmetic mean, a radio-weighted average. Using the radio luminosity $P_{i}$, we computed the radio-weighted average of a quantity $Q$ as

$$
\langle Q\rangle_{\mathrm{obbs}_{\mathrm{s}}}=\sum_{i}\left(Q_{i} P_{i}\left(v_{\mathrm{obs}}\right)\right) / \sum_{i} P_{i}\left(v_{\mathrm{obs}}\right) \text {. }
$$

The sum is taken across all radio-emitting cells that lie along the line of sight $(\mathrm{LoS})$. As the total emission volume changes with frequency, the radio-weighted average $\langle Q\rangle_{v_{\text {obs }}}$ also depends on the observing frequency $v_{\text {obs. }}$. In Appendix B, we describe how we take the cyclic property of angles into account when averaging angles.

\subsection{Polarization}

We compute the integrated polarized emission of our radio relic following the formalism of Burn (1966):

$P_{\mathrm{pol}}\left(\lambda^{2}\right)=\frac{\sum_{\text {los }} P_{\mathrm{tot}} \Pi \exp \left(2 i\left(\epsilon_{\mathrm{int}}+\mathrm{RM} \lambda^{2}\right)\right) \mathrm{d} s}{\sum_{\text {los }} P_{\mathrm{tot}} \mathrm{d} s}$,

using the emission per volume in each simulation cell $P_{\text {tot }}$, the intrinsic degree of polarization $\Pi$ and the intrinsic angle of polarization $\epsilon_{\text {int }} . R M \lambda^{2}$ accounts for Faraday rotation. The intrinsic degree of polarization $\Pi$ at observing frequency $\nu_{\text {obs }}$ is computed using the parallel and perpendicular components of the radio emission, equations (3) and (4) (e.g. Rybicki \& Lightman 1986):

$\Pi=\frac{P_{\|}-P_{\perp}}{P_{\|}+P_{\perp}}$.

The intrinsic angle of polarization, $\epsilon_{\text {int }}$, is computed with respect to the horizontal axis of the projected maps. Each simulation cell can be considered to be filled with a uniform magnetic field and, in this case, the intrinsic angle of polarization is perpendicular to the direction of the projected magnetic field. If the emission is going through a magnetized medium, the intrinsic angle of polarization is Faraday rotated. In equation (6), RM $\lambda^{2}$ determines the amount of Faraday rotation. Here, $\lambda$ is the wavelength corresponding to the observation frequency and RM is the rotation measure (RM) of the ambient medium. The RM at a distance $x$ from the observer is computed as:

$\mathrm{RM}=812 \int_{0}^{x} \frac{n_{\mathrm{e}}}{10^{-3} \mathrm{~cm}^{-3}} \frac{B_{\mathrm{para}}}{\mu \mathrm{G}} \frac{\mathrm{d} l}{\mathrm{kpc}}\left[\frac{\mathrm{rad}}{\mathrm{m}^{2}}\right]$.

The integral is taken along the LoS. $n_{\mathrm{e}}$ and $B_{\text {para }}$ are the thermal electron number density and parallel magnetic field component, respectively, along the LoS. Faraday rotation occurs either outside of the emitting region, external Faraday rotation, or inside the source, internal Faraday rotation.

The summation of equation (6) provides a complex number, from which the polarization angle ( $E$-vector) is computed as:

$\epsilon_{\mathrm{pol}}\left(\lambda^{2}\right)=\frac{1}{2} \arctan \left(\frac{\operatorname{Im}\left(P_{\mathrm{pol}}\left(\lambda^{2}\right)\right)}{\operatorname{Re}\left(P_{\mathrm{pol}}\left(\lambda^{2}\right)\right)}\right)$.

We additionally compute the angle of the $B$-vector:

$\beta_{\mathrm{pol}}\left(\lambda^{2}\right)=\epsilon_{\mathrm{pol}}\left(\lambda^{2}\right)-90^{\circ}$. 
We note that the $B$-vector only corresponds to the magnetic field direction in the case of a uniform field and without Faraday rotation. In a more complex situation, it is only a measure for the observed polarization angle.

Several effects can reduce the degree of polarization (e.g. Tribble 1991; Sokoloff et al. 1998; Arshakian \& Beck 2011). In an extended source, the polarized emission emitted at the far side will undergo a different amount of Faraday rotation than that emitted at the near side, leading to depolarization. Also, instrumental effects can cause depolarization. Beam depolarization occurs if different polarization orientations lie within the same telescope beam and hence annul each other partly. Other instrumental effects, such as bandwidth depolarization, can depolarize the emission. We have not included any of those effects and we direct readers to textbooks such as Klein \& Fletcher (2015) and references therein for more information.

\section{RESULTS}

\subsection{Simulated emission from radio relics}

So far, only Skillman et al. (2013) and Wittor et al. (2017) have studied the properties of magnetic fields in radio relics in cosmological simulations. The simulation in the present work has an unprecedented numerical resolution, which is necessary to evolve a small-scale dynamo (see Vazza et al. 2018b for details), giving us a plus for computing the polarized emission in radio relics.

In this work, we focus on the re-simulation of a $\sim 10^{15} \mathrm{M}_{\odot}$ galaxy cluster that undergoes a major merger at $z \approx 0.2$, producing two powerful shock waves. In Fig. 1, we plot the gas density overlaid with radio-emission contours at $v_{\mathrm{obs}}=1.4 \mathrm{GHz}$ projected along the three different axes of the simulation box. The edge-on view shows two prominent, large-scale shock waves that produce radio emission on a $\sim$ Mpc scale. For our analysis, we focused on the larger and brighter of the two (see blue arrows in Fig. 1). For the analysis, we only included those cells that lie in the area of $(1580 \times 790) \mathrm{kpc}^{2}$ around the relic (i.e. see top panel in Fig. 3). If the same relic is observed along the two other orthogonal LoS, then the radio emission becomes more extended and dimmer. In the remainder of this paper, we will refer to the relic as it is observed in the three different projections: "edgeon', 'face-on' and 'side-on' as labelled in Fig. 1. In the following, we will compare the typical quantities of the simulated relic to observations.

The radio relic in our simulation is at a distance of $\sim 1.7 \mathrm{Mpc}$ to the centre of mass of the cluster (see Table 2). Its apparent morphology varies significantly with the projection. When observed edge-on, it has a small surface that is thin and elongated, while, when observed both face-on and side-on, it shows much larger surfaces and more filamentary structures. The radio power of the relics is of the order of $\sim 10^{30} \mathrm{erg} \mathrm{s}^{-1} \mathrm{~Hz}^{-1}$ at $1.4 \mathrm{GHz}$. The small discrepancies between the different projections are due to the emission in front or behind the relic along the LoS. The simulated relic lies below the massluminosity relation derived by de Gasperin et al. (2014), but one has to take into account that this relation has been derived using a much more powerful class of double radio relics and that it might be biased towards brighter objects due to the sensitivity of radio telescopes. Still, the simulated relic is fainter than most observed single radio relics. This could be a consequence of either the low Mach number or the significantly low magnetic field strength at the relic's position.
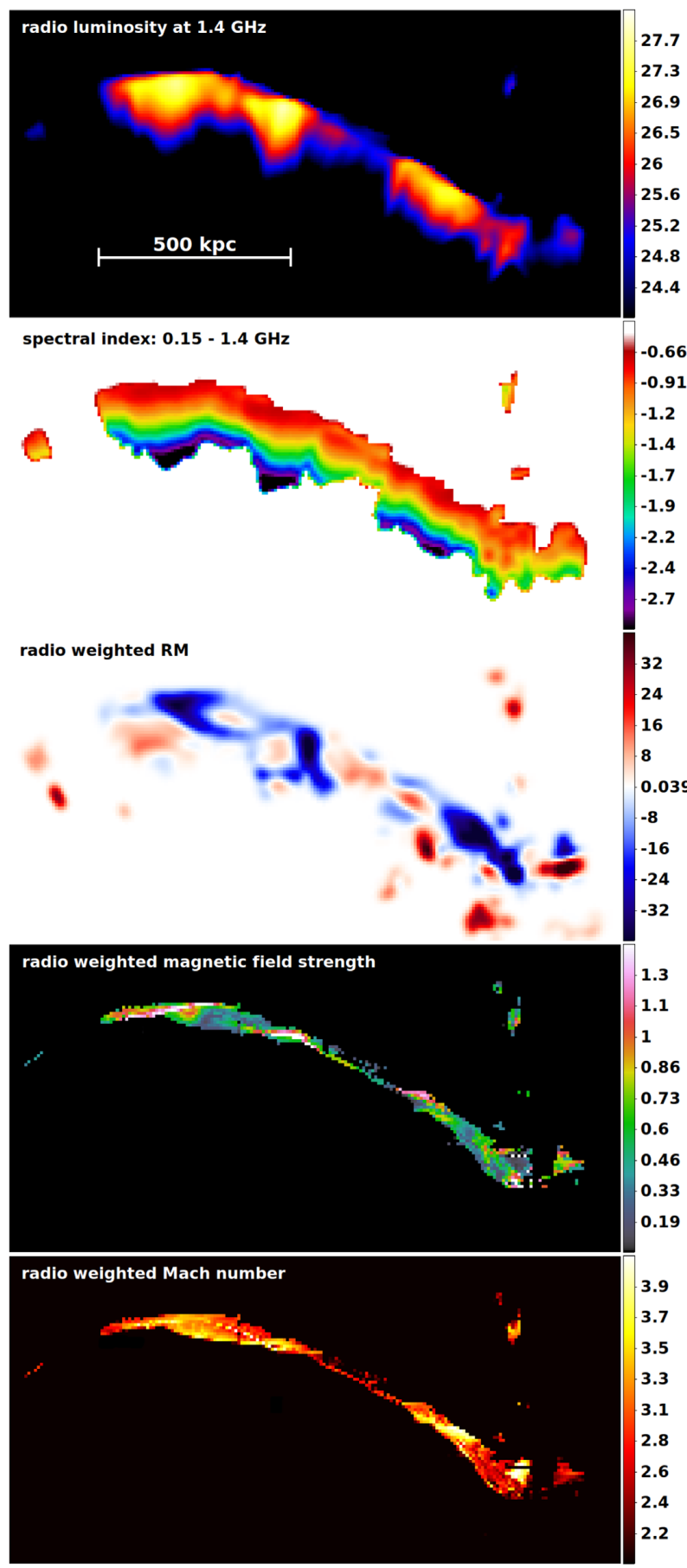

Figure 3. Physical quantities at the relic's position. From top to bottom: radio luminosity in $\log _{10}\left(\mathrm{erg} \mathrm{s}^{-1} \mathrm{~Hz}^{-1}\right)$, spectral index between $0.15 \mathrm{GHz}$ and $1.4 \mathrm{GHz}$, radio-weighted $\mathrm{RM}$ in $\mathrm{rad} \mathrm{m}^{-2}$ as well as the radio-weighted magnetic field strength in $\mu \mathrm{G}$ and radio-weighted Mach number at the shock surface. In all cases, the radio weighting was done at $1.4 \mathrm{GHz}$. (A colour version is available in the online article.)

For several observed radio relics, the Mach number derived from radio observations is larger than that derived from X-rays (e.g. Hong, Kang \& Ryu 2015; Hoang et al. 2018, and references therein). This discrepancy could be related to projection effects (Hong et al. 2015) or systematic errors in the X-ray observations (Akamatsu et al. 2017). Hence, we calculated the Mach number distribution across the shocked cells that have $M \geq 2$ (see top panel 
Table 2. The table shows the properties of our simulated radio relic. From left to right: the projection, the projected relic surface area, the radio luminosity at $1.4 \mathrm{GHz}$, the average Mach number (for Mach numbers >2), the Mach number computed using the spectral index between $0.15 \mathrm{GHz}$ and $1.4 \mathrm{GHz}$, the average and radio-weighted average magnetic field strength at the shock front, and the largest linear size. The radio weighting was done at $1.4 \mathrm{GHz}$.

\begin{tabular}{lccccccc}
\hline projection & $S\left[10^{3} \cdot \mathrm{kpc}^{2}\right]$ & $P_{1.4 \mathrm{GHz}}\left[10^{30} \mathrm{erg} \mathrm{s}^{-1} \mathrm{~Hz}^{-1}\right]$ & $\langle M\rangle$ & $M_{(0.15-1.4) \mathrm{GHz}}$ & $\langle B[\mu \mathrm{G}]\rangle$ & $\langle B[\mu \mathrm{G}]\rangle 1.4 \mathrm{GHz}$ & $\mathrm{LLS}[\mathrm{Mpc}]$ \\
\hline edge-on & 226 & 1.29 & 2.45 & 3.54 & 0.20 & 0.56 \\
face-on & 421 & 1.32 & 2.33 & 3.44 & 0.40 & 0.64 \\
side-on & 248 & 1.30 & 2.37 & 3.48 & 0.28 & 0.35 \\
\hline
\end{tabular}

in Fig. 4). Additionally, we computed the integrated radio spectral index ${ }^{4}$ for each projection, and the corresponding Mach number ${ }^{5}$ between $1.4 \mathrm{GHz}$ and $0.15 \mathrm{GHz}$. The spectral index obtained for each projection is $\alpha_{\mathrm{R}} \approx-1.17$ corresponding to Mach numbers of $M \approx 3.5$. Therefore, the Mach number derived from the spectral index is much larger than most of the Mach numbers in the simulation (see red line in Fig. 4). These findings are in agreement with Ha, Ryu \& Kang (2018), where they found that Mach numbers derived from radio observations are biased towards larger values because the shock acceleration efficiency strongly depends on the Mach number.

The average Mach number and average magnetic field strength are given in Table 2, where we additionally show their radioweighted averages (see equation 5). In the remainder of the paper, we will discuss the other properties of the simulated relic and we will focus mainly on the edge-on view. We choose the edge-on view because the relic morphology is similar to the assumed 'prototype' of radio relics and because the direction of the shock normal is well defined (see. Fig. 5). Throughout the work, we will highlight differences between and similarities with the other two LoS.

\subsection{Distribution of magnetic fields}

The maps of projected gas density, temperature and magnetic field strength at the relics position are shown in Fig. 6. We can observe that the upstream density and temperature are very regular at the top-left edge of the relic. Yet at the lower-right edge, a sub-clump is falling into the cluster, causing this sector to be disturbed. The upstream magnetic field shows a similar topology, while we observe some small-scale fluctuations in the downstream. Finally, we can observe that the shock front is not uniform as it is highlighted better in the temperature map.

Fig. 4 shows the magnetic field strength's distribution of the radio-emitting cells. Most of the radio-emitting cells have magnetic field values that are smaller than $1 \mu \mathrm{G}$ and only a few cells have magnetic field strengths above $2 \mu \mathrm{G}$. The average magnetic field strength producing the radio relic is in the range of $0.2-0.4 \mu \mathrm{G}$, while the radio-weighted magnetic field strength is $\sim 1.05 \mu \mathrm{G}$. These magnetic field values are smaller than estimations at the position of observed relics (see fig. 14 in Bonafede et al. 2013). This occurs in our simulation since the small-scale dynamo probably did not have sufficient time to evolve and amplify the magnetic field at the relic's position.

In Fig. 5, we show the shock normal as well as the radio-weighted (see equation 5) orientation of the projected magnetic field at $1.4 \mathrm{GHz}$ in order to get a sense of the magnetic field behaviour. The radio-weighted magnetic field direction changes rapidly across

$$
\begin{aligned}
{ }^{4} \alpha_{\mathrm{R}} & =\frac{\log _{10}\left(P_{1.4} / P_{0.15}\right)}{\log _{10}\left(v_{1.4} / \nu_{0.15}\right)} \\
{ }^{5} \alpha_{R} & =\frac{2 M^{2}+2}{2 M^{2}-2}
\end{aligned}
$$
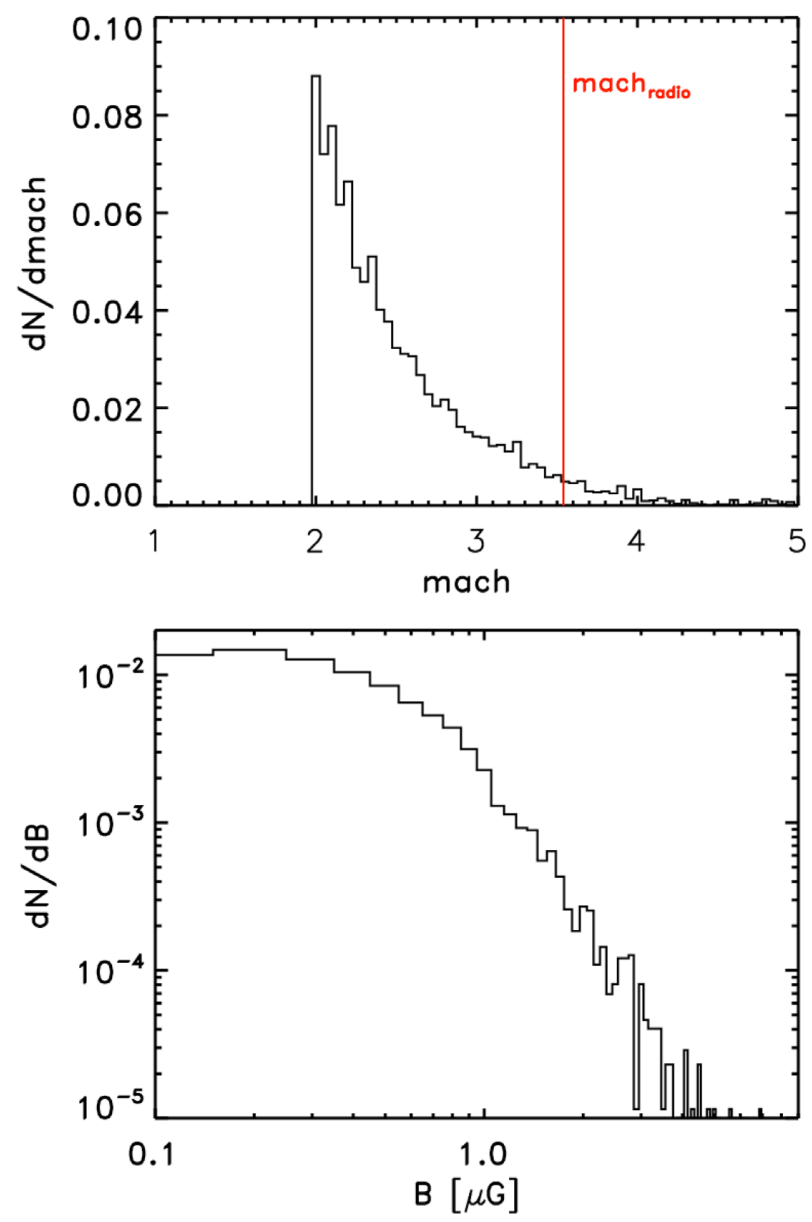

Figure 4. Volumetric distributions of Mach number (top) and magnetic field strengths (bottom) of radio-emitting cells at $1.4 \mathrm{GHz}$. The distributions have been normalized to the number of cells that emit in radio. The red line in the top plot marks the Mach number computed from the spectral index between $1.4 \mathrm{GHz}$ and $0.15 \mathrm{GHz}$.

the length of the relic. We can observe that the magnetic field aligns with the shock surface in several regions, while it is perpendicular to the shock surface in others.

We also compute the magnetic power spectrum and its correlation length, i.e. the outer scale of the spectrum, in the $(7.9 \times 200 \mathrm{kpc})^{3}$ sub-box centred on the relic. We obtained a correlation length of $\sim 248.8 \mathrm{kpc}$, by fast Fourier-transforming the three-dimensional magnetic field and by fitting its power spectrum with the functional form derived in Domínguez-Fernández et al. (2019). We refer the reader to Appendix $\mathrm{C}$ for more information on the magnetic spectrum.

In Fig. 3, we show different properties of the radio relic itself. First, we show the radio emission at $1.4 \mathrm{GHz}$ and the spectral index map computed between $0.15 \mathrm{GHz}$ and $1.4 \mathrm{GHz}$. The spectral index 

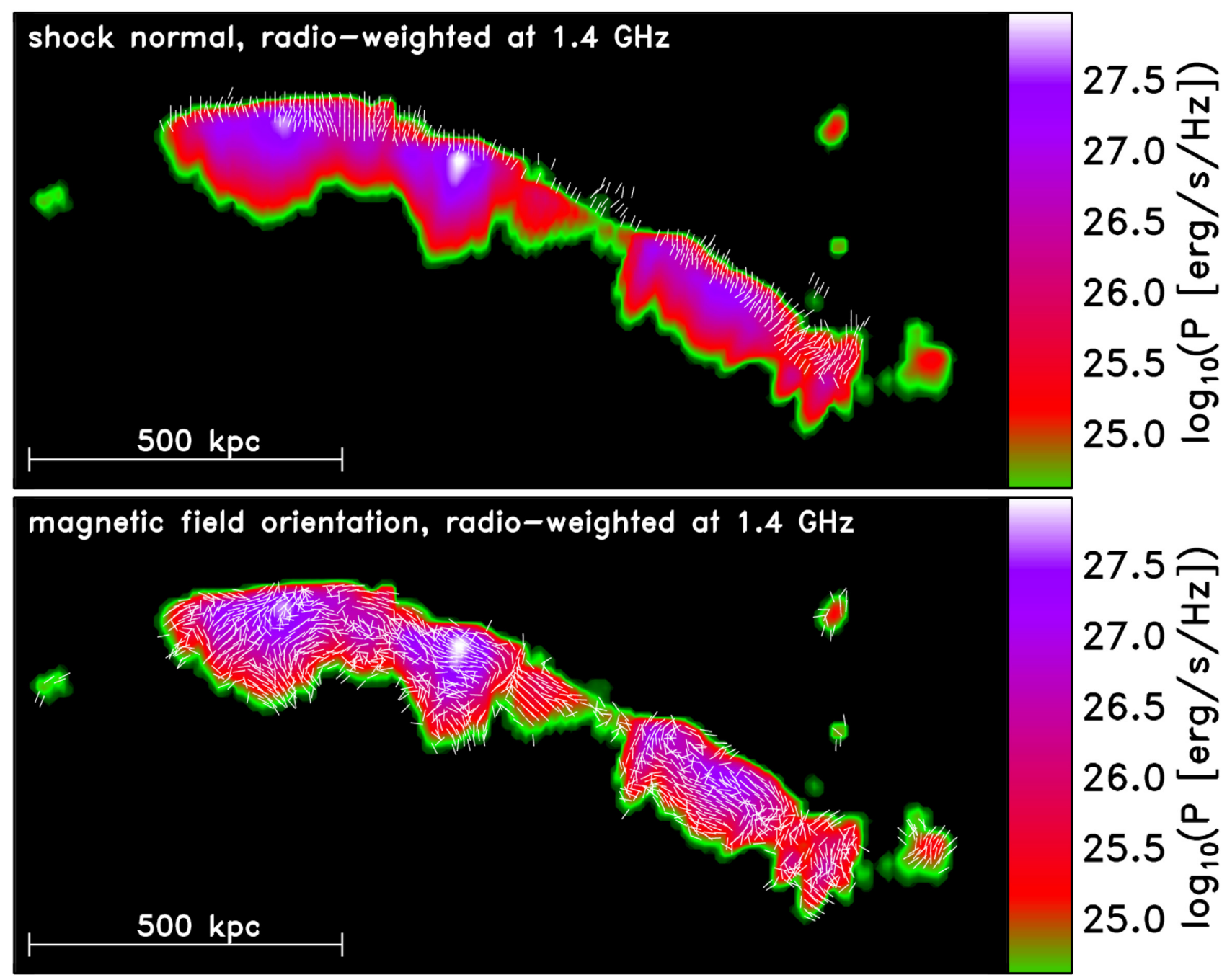

Figure 5. Radio luminosity (colour) overlaid with the radio-weighted projected shock normal (top) and the radio-weighted orientation of the physical magnetic field (bottom). The vectors have been normalized to unity.

is computed by superimposing different radio downstream profiles and hence the ageing of the spectral index is slower than expected from Fig. 2. Next, we show the radio-weighted (see equation 5) RM at the relic's position, the radio-weighted magnetic field strength and the radio-weighted Mach number at the shock front. The radio emission varies significantly across the relic, and two bright patches are visible. The latter connect via a fainter and filamentary bridge and they are located in the regions of strong Mach numbers and of large magnetic field values. We can also observe that the RM is not uniform across the relic due to the varying magnetic field strengths and densities along the LoS.

\subsection{Distributions of rotation measures}

In the presence of magnetic fields, the polarization vectors undergo Faraday rotation. The amount of rotation depends on both the strength and distribution of the RM along the LoS. As the RM distribution is most likely not uniform for an extended source, the polarization vectors will rotate differently and therefore this could enhance depolarization (see Section 2.3). In the near future, RM measurements will become more precise due to the increasing resolution of telescopes and the application of RM synthesis (e.g. Johnston-Hollitt et al. 2015; Bonafede et al. 2015; Brentjens \& de Bruyn 2005), and polarization studies will improve.

In the following, we will describe the RM distribution in our simulation in greater detail. We obtain two different RM distributions for each projection, since equation (6) can be integrated from different sides of the computational domain. We will refer to these cases as 'along LoS' and 'along -LoS'. Including the case without Faraday rotation (RM $=0$ in equation 6$)$, this gives a total of nine (independent) test cases to study the polarization of the radio relic.

First, we compute the distribution of RM found in the radioemitting cells. As the surface and the depth of the emitting region change with frequency, the RM distribution depends on the observing frequency as well. In the top panels of Fig. 7, we show the normalized RM distributions at $1.4 \mathrm{GHz}$, where we consider only values of $\mathrm{d} N / \mathrm{dRM}>1$ per cent. In the bottom panels of Fig. 7, we plot the average and standard deviation of each distribution for different frequencies.

The standard deviations for the edge-on and side-on views are a few tens $\mathrm{rad} \mathrm{m}^{-2}$ and they remain fairly constant with frequency. On the other hand, when seen face-on, the standard deviation is one 


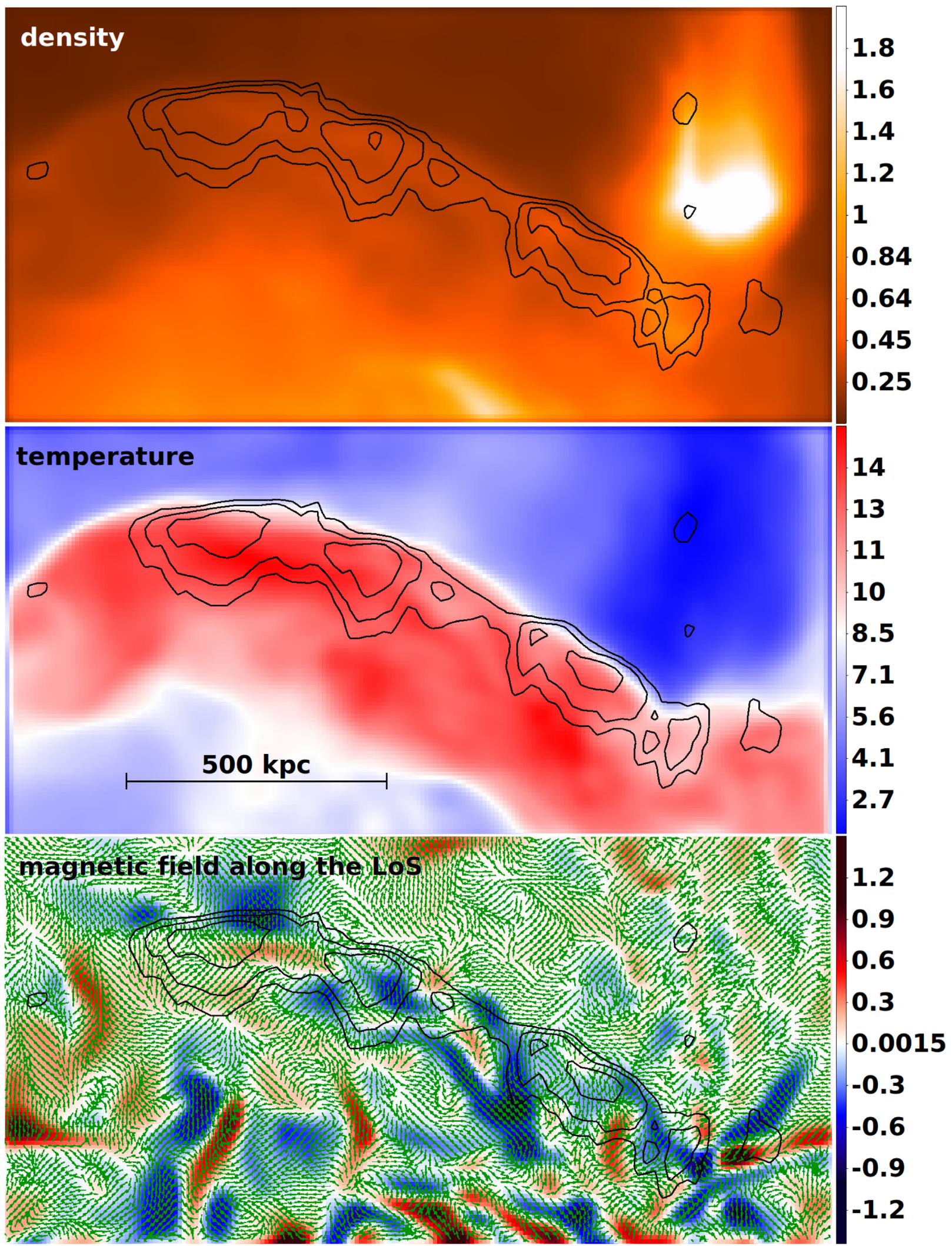

Figure 6. Close-up view of the relic region. The black contours show the radio power at $1.4 \mathrm{GHz}$ at $4 \times\left[10^{24}, 10^{25}, 10^{26} 10^{27}\right] \mathrm{erg} \mathrm{s}^{-1} \mathrm{~Hz}^{-1}$. Top: projected density in $10^{-27} \mathrm{~g} \mathrm{~cm}^{-3}$, middle: projected temperature in $10^{7} \mathrm{~K}$ and bottom: slice through the magnetic field component along the line of sight in $\mu \mathrm{G}$ (colour) overlaid with the magnetic field vectors (green vectors) in the plane. The vectors are scaled to unity. (A colour version is available in the online article.) 

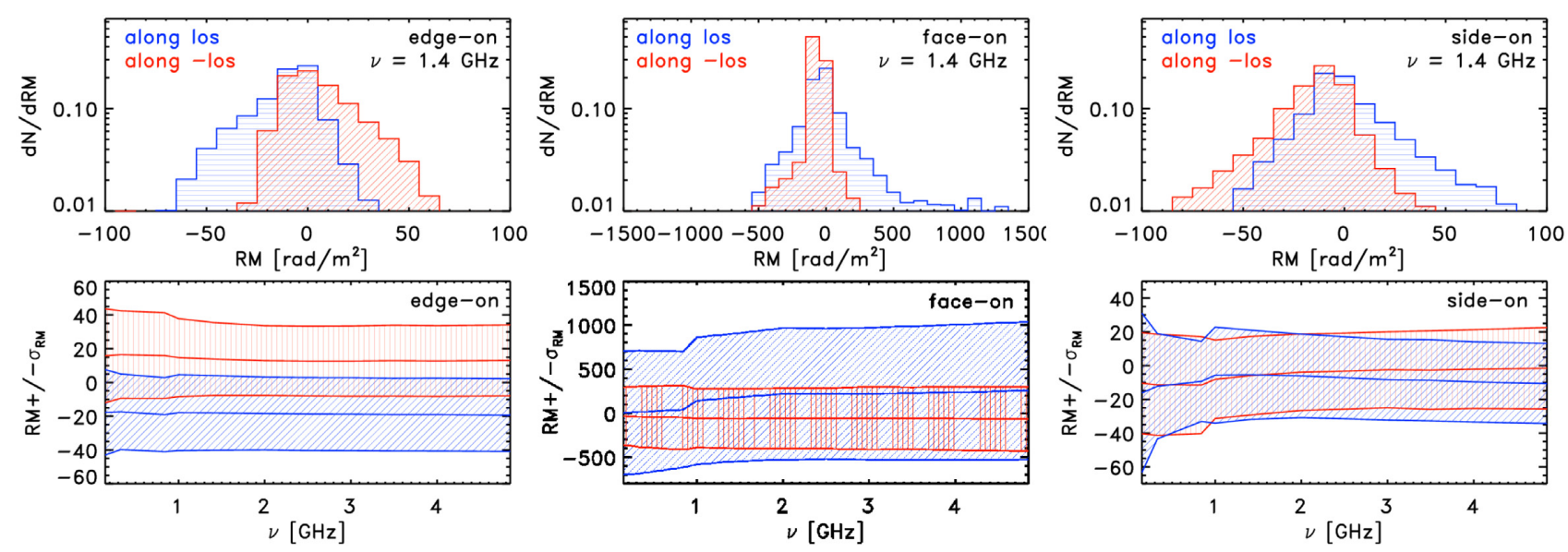

Figure 7. Normalized distributions of the rotation measures in the radio-emitting cells for the edge-on, face-on and side-on views (left to right). We only show the distributions where $\mathrm{d} N / \mathrm{dRM}>1$ per cent. The top row shows the distributions of the radio-emitting cells at $1.4 \mathrm{GHz}$ for each projection (the different range of the $x$-axis in the central panel should be noted). The bottom panels show the average values (in $\left[\mathrm{rad} \mathrm{m}^{-2}\right]$ ) plus/minus the one standard deviation of the distributions as a function of frequency. The colours match the colours of the distribution: blue (dash-dotted) along the LoS and red (dotted) along the LoS but in the opposite direction. (A colour version is available in the online article.)

order of magnitude higher. In this face-on view, the distributions are much broader and show extended tails with values above $\pm 200 \mathrm{rad} \mathrm{m}^{-2}$ for at least one viewing direction. The relic is behind the cluster in this view (see Fig. 1), and so the emission must pass through a longer magnetized region.

The average values of the different distributions remain almost constant at high frequencies, while their variation becomes larger at low frequencies. These variations are still within one standard deviation. The fact that we obtain less variation at high frequencies might have a numerical nature as the cooling regions at these frequencies are under-resolved. The effective spatial resolution of any hydro-MHD scheme ( $32 \mathrm{kpc}$ in our simulation) is coarser than the nominal one, and therefore we cannot account for any variation of the RM on smaller scales that become more important at higher frequencies.

Summarizing the RM analysis, we obtain standard deviations of the RM distributions of a few $10 \mathrm{rad} \mathrm{m}^{-2}$ in most cases of the simulation, and values between $\sim 300-800 \mathrm{rad} \mathrm{m}^{-2}$ only if the radio emission originates from behind the cluster.

In the following, we want to examine whether or not the RM in our simulation agrees with the RM observed in the ICM that can be obtained through the polarization analysis of background sources: Hammond, Robishaw \& Gaensler (2012) compiled an RM catalogue of extragalactic sources located at redshift $0<z$ $<5.3$. They found that the variance of the RM distributions does not change with redshift and they estimated a standard deviation of $\sigma_{\mathrm{RM}} \approx 23 \mathrm{rad} \mathrm{m}^{-2}$; Böhringer, Chon \& Kronberg (2016) estimated the RM dispersion in regions located more than $1 \mathrm{Mpc}$ away from the cluster centre of a large sample of galaxy clusters, finding a value of $\approx(57 \pm 6) \mathrm{rad} \mathrm{m}^{-2}$; Johnston-Hollitt (2004) studied the RM of the north-western relic in Abell 3667 and found that locally the values can be $\sim-165.1 \mathrm{rad} \mathrm{m}^{-3}$ and $\sim 98.2 \mathrm{rad} \mathrm{m}^{-2}$. Yet, higher-resolution studies of the RM distribution showed that the dispersion can be smaller at the relic; Bonafede et al. (2013) measured a dispersion of $\sim 5.2 \mathrm{rad} \mathrm{m}^{-2}$ for the Coma cluster. Consequently, we conclude that the RM and its variation produced in our simulation agrees with current observations. Yet for higher magnetic field values, we would need a correspondingly smaller correlation length in order to recover the same $\sigma_{\mathrm{RM}}$ (the correlation lengths of the magnetic field in the whole simulation box are $\sim 250-300 \mathrm{kpc}$ ).

We have only used the $\sigma_{\mathrm{RM}}$ of either the cluster periphery or the entire cluster, and any contribution of the intergalatic medium (e.g. integrating over $\sim 800 \mathrm{Mpc}$ for $z \approx 0.2$ ) would have to be added. We do not include them here as these contributions are small and they would not change our results: Akahori \& Ryu (2010, 2011) obtained a contribution of about $\sim 1 \mathrm{rad} \mathrm{m}^{-2}$ for filaments and an RM saturation of $\sim 7-8 \mathrm{rad} \mathrm{m}^{-2}$ for $z \geq 1$. Furthermore, they showed that the main contributors to the RM are galaxy clusters. These results were also confirmed by Vazza et al. (2018a), who also argued that the intergalactic RM contribution should be even smaller in the case of purely astrophysical magnetic seed fields.

As a final caution, one should notice that the RM distributions measured here are neither Gaussian nor symmetric (see Fig. 7). Yet, several works (e.g. Burn 1966; Tribble 1991) assume Gaussian or symmetric RM distributions, which can lead to systematic effects in the inference of magnetic fields.

\subsection{The intrinsic properties of the polarized emission}

We computed the integrated polarized emission (equation 6) and the corresponding $E$-vector (equation 10). As an example, we plot the fractional polarization overlaid with the corresponding $E$-vectors for two different observing frequencies in the last two panels of Fig. 8. Additionally, we show the radio-weighted, at $4.85 \mathrm{GHz}$, orientation of the physical electric wave to visualize its characteristic orientation. Locally, the $E$-vectors have the same orientation, but they do not show alignments on scales above $500 \mathrm{kpc}$. Also, there is no strong correlation between the direction of the $E$-vectors and the shock normal (compare with Fig. 5). The orientation of the $E$-vectors seen edge-on starts to randomize locally for $v<1.4 \mathrm{GHz}$. On the other hand, at $v=4.85 \mathrm{GHz}$ the effect of Faraday rotation is small and we observe an orientation similar to the case without Faraday rotation. Along each LoS, a few bright cells dominate the radio emission. Hence, their intrinsic degrees of polarization determine the polarization fraction that does not significantly decrease with frequency. In these maps, 

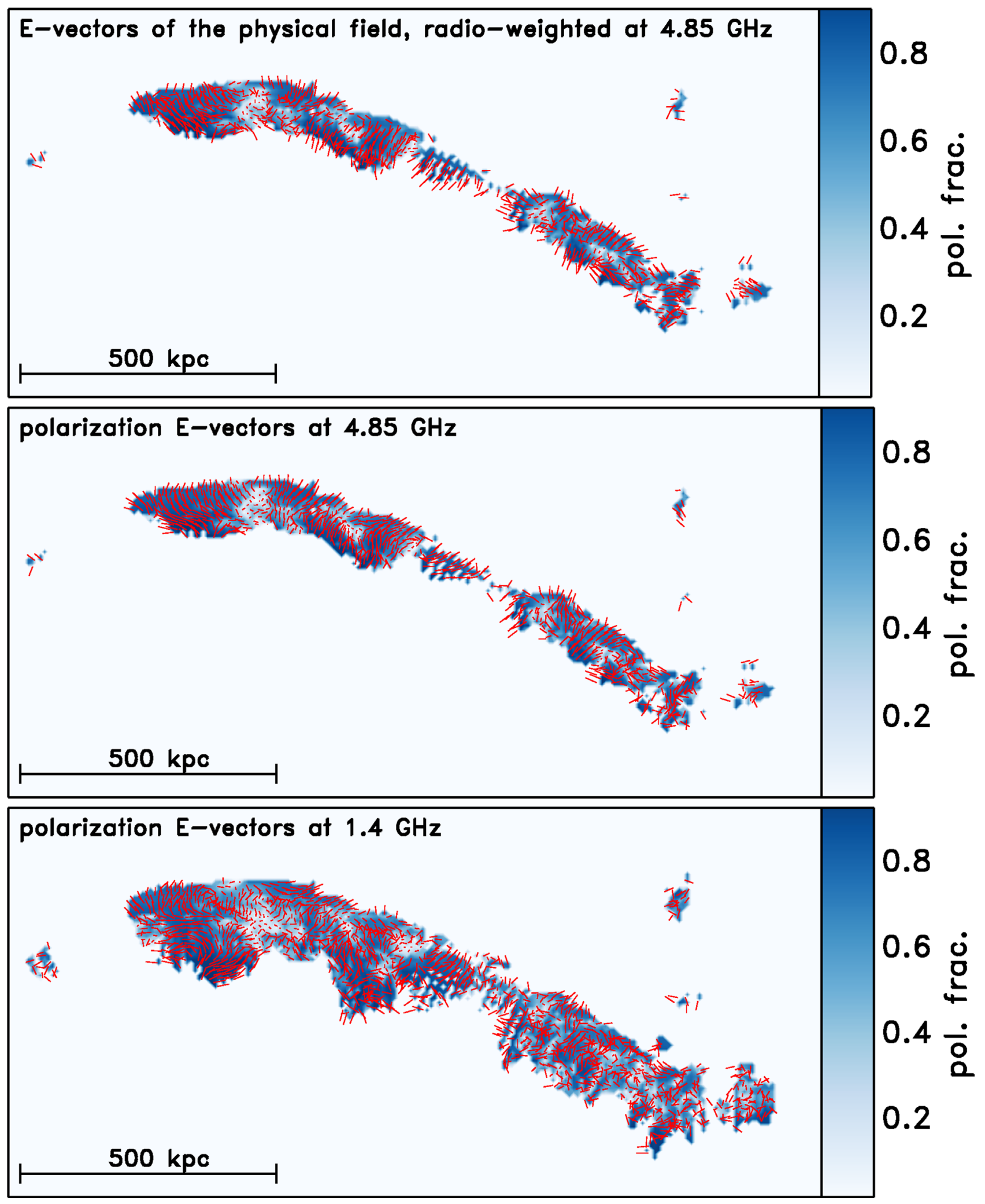

Figure 8. Projected fractional polarization (colour) overlaid with the $E$-vectors (white vectors). The top row shows the radio-weighted $E$-vectors of the physical field at $v=4.85 \mathrm{GHz}$. The middle row and the last row show the polarization $E$-vectors at $v=4.85 \& 1.4 \mathrm{GHz}$. The vectors have been normalized to unity. (A colour version is available in the online article.) 

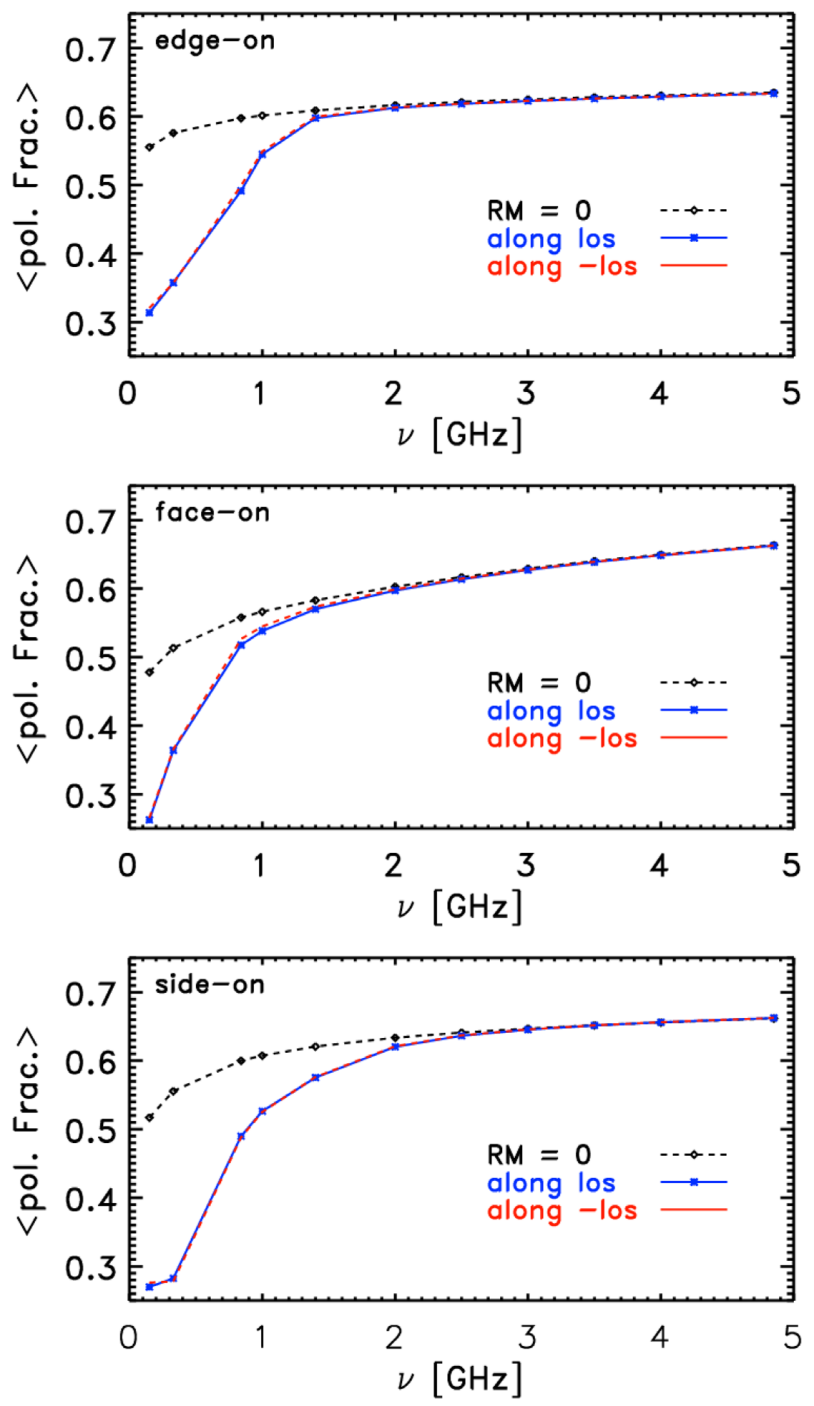

Figure 9. Radio-weighted degree of polarization. The different panels display the results for the different projections: edge-on, face-on and side-on (top to bottom). The different lines give the different RM models. (A colour version is available in the online article.)

we do not apply any observational cut and, hence, the extent of the polarization fraction into the downstream mirrors the extent of the surface brightness, which is larger for lower frequencies. Since the intrinsic degree of polarization increases for steeper spectra, the polarization fraction becomes larger in the downstream.

We do not show the $E$-vectors if the relic is seen along the opposite direction of the LoS, as the pattern is similar. In both the face-on and side-on views, the orientation of $E$-vectors is similar to the edge-on view. However, their morphology is more complex and cannot be directly related to the true shock normal.

As observations tend to pick up the brighter parts of the relic, we computed the radio-weighted average degree of polarization for frequencies in the range from $150 \mathrm{MHz}$ to $4.85 \mathrm{GHz}$. In Fig. 9, we plot the degrees of polarization versus frequency. We also include the control cases $\mathrm{RM}=0$ that reflect the intrinsic degree of polarization. We note that we computed the RM on scales of the grid resolution and any higher-amplitude small-scale structures in the Faraday depth, due to the finite resolution of our simulation, are neglected. Therefore, the degree of polarization reflects depolarization by internal Faraday rotation, while external Faraday rotation only rotates the polarization vectors without causing any additional depolarization.

Independent of the projection and the RM selection, the degree of polarization is always larger at high frequencies, i.e. $\sim 0.64$ seen edge-on and $\sim 0.67$ seen face-on and side-on, and decreases for lower frequencies. The degree of polarization drops to $\sim 0.31$ seen edge-on and $\sim 0.26-0.28$ seen face-on and side-on at $150 \mathrm{MHz}$ for the cases that include Faraday rotation. Without Faraday rotation, the degree of polarization is significantly higher at $150 \mathrm{MHz}$, i.e. $\geq 48$ per cent. The degree of polarization for the different test cases including $\mathrm{RM}$ converges with the control case, i.e. $\mathrm{RM}=0$, above 1.0-2.0 GHz.

One would expect that the face-on projection (which features the highest values of RM) would show the lowest degree of polarization and the slowest convergence to the control case of $\mathrm{RM}=0$ due to the $\exp \left(-\mathrm{RM}^{2} \lambda^{4}\right)$ factor. Nevertheless, this is not the case because the extent of the relic along the LoS is much shorter than in the other two cases, i.e. $\sim 160 \mathrm{kpc}$ instead of $\geq 400 \mathrm{kpc}$. Therefore, the face-on projection effectively probes fewer polarization vectors and depolarization since the internal Faraday rotation is small.

In all three control cases, i.e. $\mathrm{RM}=0$, the degree of polarization decreases at lower frequencies. The downstream width of the relic is larger at low frequencies and, hence, polarization is also probed at a larger distance to the shock front. The farther into the downstream, the more magnetic fluctuations we encounter (see Fig. 6) and, as a consequence, the orientation of the intrinsic angle of polarization is more random. This is also illustrated at low frequencies. In summary, we obtain an average degree of polarization $\geq 50$ per cent for $v \geq 1.0-1.4 \mathrm{GHz}$ and it is significantly larger than what it is obtained in observations (see Table 1). On the other hand, our results are in line with previous ENZO simulations by Skillman et al. (2013), who measured a maximum degree of polarization $\sim 75$ per cent. We reiterate that the degree of polarization only accounts for depolarization due to internal Faraday rotation and, thus, including the beam and/or bandwidth depolarization can decrease it.

\subsection{Synthetic radio observations}

In order to compare with real radio observations, we performed synthetic observations of our cluster with the Effelsberg telescope, the VLA and LOFAR-HBA. After locating our relic at different redshifts and converting the emitted power into the physical frame, we convolved the luminosity and the integrated polarized emission that were previously finely sampled (see equations 1 and 6 respectively) to the proper resolution/beam size of the assumed telescope configurations, $d_{\text {beam }}$. The detection threshold, $I_{T}$, is computed as a flux density level of three times the noise per beam, $\sigma_{\text {noise }}: I_{T}=3 \times$ $\sigma_{\text {noise }} /\left(1.1333 \cdot d_{\text {beam }}\right)^{2}$.

We considered the fiducial parameters shown in Table 3, which were taken from recent papers. In particular, we use the parameters given in table 1 of Shimwell et al. (2017) for the directionindependent LOFAR Two Meter Sky Survey for the LOFAR-HBA mock observation. We use the parameters in table 2 of Rajpurohit et al. (2018) for the VLA mock observation and those in table 3 of Kierdorf et al. (2017) for the Effelsberg mock observations. In the case of the Effelsberg mock observation, we place the relic at $z=0.1$ and 0.2 , as the large beam would cover more than the entire simulation box for larger redshifts. On the other hand, for the LOFAR-HBA and VLA mock observation, we have to use 
Table 3. Fiducial parameters for our mock observations. The first two columns give the name of the telescope and the observing frequency. The third, fourth and fifth columns provide the beam size, thermal noise and estimated detection threshold respectively. The last two columns give the observed polarization fraction of the relic at $z=0.4(z=0.1$ for Effelsberg) and $z=0.5(z=0.2$ for Effelsberg) respectively. The parameters have been taken from table 1 in Shimwell et al. (2017), table 2 in Rajpurohit et al. (2018) and table 3 in Kierdorf et al. (2017).

\begin{tabular}{lcccccc}
\hline Telescope & $v[\mathrm{GHz}]$ & $d_{\text {beam }}[\operatorname{arcsec}]$ & $\sigma_{\text {noise }}\left[\mu \mathrm{Jbeam}^{-1}\right]$ & $I_{T}\left[\mu \mathrm{J} \operatorname{arcsec}^{-2}\right]$ & pol. frac. $[\%]$ at $z=0.4 / 0.1$ & pol. frac. $[\%]$ at $z=0.5 / 0.2$ \\
\hline LOFAR & 0.15 & 25 & 500 & 2.1 & 5.7 & 4.8 \\
VLA & 1.4 & 5 & 6 & 0.64 & 40.4 & 47.2 \\
VLA & 1.4 & 7 & 8 & 0.43 & 39.6 & 31.2 \\
VLA & 1.4 & 11 & 12 & 0.26 & 26.0 & 38.8 \\
VLA & 1.4 & 16 & 18 & 0.18 & 16.1 & 8.1 \\
VLA & 1.4 & 25 & 26 & 0.11 & 0.083 & 8.6 \\
Effelsberg & 4.85 & 159 & 800 & &
\end{tabular}

$z=0.4$ and 0.5 , as they would have a higher physical resolution than our reconstructed mesh at smaller redshifts. Only a few polarization studies using LOFAR at $v \sim 0.15 \mathrm{GHz}$ have been published so far (e.g. O'Sullivan et al. 2019), but none concerning radio relics. Therefore, we only include these mock observations as a sanity test for our implementation. In the following, we neglect any sort of bandwidth depolarization (e.g. Hamaker, Bregman \& Sault 1996; Sault, Hamaker \& Bregman 1996). We provide the radio-weighted degrees of polarization for each mock observation in Table 3.

The degree of polarization of the Effelsberg observation is 16.1 per cent at $z=0.1$ and 8.6 per cent at $z=0.2$. Beam depolarization occurring in the large telescope beam, $\sim(159 \mathrm{arcsec})^{2}$ corresponding to $(64 \mathrm{kpc})^{2}$ and $(112 \mathrm{kpc})^{2}$ at $z=0.1$ and 0.2 , respectively, produce these small values. These results agree with the 5 per cent polarization in Abell $1612(z \approx 0.172)$ reported by Kierdorf et al. (2017) who used the Effelsberg telescope to study the polarization of four radio relics at 4.85 and $8.35 \mathrm{GHz}$. Yet, our results are at odds with their measurements of 36 per cent and 15 per cent for the relics in CIZA J2242.8+5301 $(z \approx 0.192)$ and in 1 RXS J0603.3+4214 $(z \approx 0.225)$.

Our LOFAR-HBA mock observations at $0.15 \mathrm{GHz}$ show a polarization degree below 6 per cent at both redshifts. We expect that this is a numerical artefact and has no physical meaning. Hence, we do not expect to detect significant polarization at LOFAR-HBA frequencies.

In Fig. 10, we plot the integrated polarized emission overlaid with the corresponding $E$-vectors for three different VLA mock observations at $z=0.4$. Furthermore, we show the degree of polarization for the different VLA configuration in Fig. 11. Our results lie in the range of 26-50 per cent and they decrease with increasing beam size, which indicates beam depolarization at both redshifts.

In order to compare with real observations, we compute first the distributions of the average and the local maximum degree of polarization of all radio relics studied in polarization at $v \approx 1.4 \mathrm{GHz}$ (see Table 1). Then, we compare these to the distribution functions from our VLA mock observations at $v=1.4 \mathrm{GHz}$ (see Table 3), and plot the two distribution functions in the bottom panel of Fig. 11. The distribution functions (average and local maximum) of the mock observations have lower dispersion and peak at higher values than the corresponding distribution functions of the real observations. Even though our final results weakly agree with polarization observations, a larger statistical sample of simulated relics is needed to test whether their properties agree with reality.

\subsection{Morphological properties}

The observed morphology of the polarized emission is quite diverse (see references in Section 1.1). Hence, our small sample cannot explore the variety given by observations. Our relic does not show an overall global alignment of $E$-vectors as observed in some radio relics. However, it shows local structures that match features observed in radio relics, so it may offer useful hints to interpret observed polarization structures. In the following, we want to highlight three cases that show qualitative similarities with observed structures.

In Fig. 12, we show a close-up view of our radio relic at $1.4 \mathrm{GHz}$ seen in the three different projections. The arrows point to three different regions that we want to further investigate: a region with locally aligned $E$-vectors (A), a bridge structure (B) and a depolarized 'brush' (C). In this section, we do not apply any detection threshold or re-binning to a specific beam size as highfrequency radio observation can indeed resolve structures of $8 \mathrm{kpc}$ or smaller in radio relics (e.g. Rajpurohit et al. 2018). We notice that the discussed radio structures are significantly larger than the effective resolution of our hydro-MHD scheme. Even if some features appear to be close to the effective resolution $(\sim 32 \mathrm{kpc})$, this is only due to the projection along the LoS, while their intrinsic three-dimensional separation is much larger.

\subsubsection{Bridge structure}

The bridge structure connects the two brighter patches of the radio relic seen edge-on. Compared to other regions, this region is very filamentary in the face-on view. In the edge-on view, the thickness of the bridge is about $\sim 150 \mathrm{kpc}$, although, in the face-on view, the filaments' extent is about $\sim 260 \mathrm{kpc}$. Hence, the vectors are probing magnetic field structures that are significantly separated. If falling within the same telescope beam, they can get depolarized. The edge-on view of the Mach number (see Fig. 3) reveals a filamentary shock structure in the bridge region. New high-resolution radio observations of relics show similar complex structures in the form of filaments and threads (e.g. Rajpurohit et al. 2018; Di Gennaro et al. 2018). Whether these structures are dominated by magnetic fields, by cosmic ray-driven small-scale instabilities, or by a complex shock morphology, cannot be established at present. The threads of the Mach number in the simulation are a product of (magneto)hydrodynamic flows and they are most likely visible if our simulated relic was observed at a higher resolution. The other parts of the relic could in principle have similar filamentary structures, but they might only appear in the bridge because there is no projection of other emissions on top.

\subsubsection{Alignment of E-vectors}

In the top-left region of the radio relic, the $E$-vectors are locally aligned to each other as seen in most radio relics. In the edge-on 
polarized intensity $[\mu \mathrm{J}$ y beam $]$

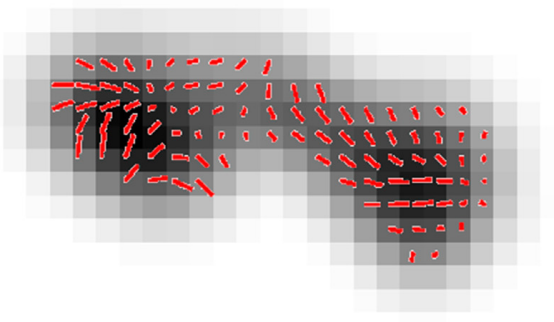

0.47

0.41

0.35

0.29

0.23

VLA $1.4 \mathrm{GHz}, z=0.4$

0.18

$\mathrm{d}_{\text {beam }}=5$ arcsec

0.12

$\sigma_{\text {noise }}=6 \mu \mathrm{Jy} /$ beam

0.058

$\mathrm{I}_{\mathrm{T}}=0.64 \mu \mathrm{Jy} /$ beam

0.38

0.34

0.29

0.25

0.21

0.17

0.13

VLA $1.4 \mathrm{GHz}, z=0.4$

0.084

$\mathbf{d}_{\text {beam }}=11$ arcsec

$\sigma_{\text {noise }}=12 \mu \mathrm{Jy} /$ beam

$\mathrm{I}_{\mathrm{T}}=0.26 \mu \mathrm{Jy} /$ beam

0.042

0.21

VLA $1.4 \mathrm{GHz}, \quad \mathrm{z}=0.4$

0.07

$\mathrm{d}_{\text {beam }}=25$ arcsec

0.046

$\sigma_{\text {noise }}=26 \mu \mathrm{Jy} /$ beam

0.023

$\mathrm{I}_{\mathrm{T}}=0.11 \mu \mathrm{Jy} /$ beam

Figure 10. VLA mock observations, at $1.4 \mathrm{GHz}$ and using beam sizes of 5,11 and $25 \mathrm{arcsec}$, of the relic placed at $z=0.4$. The polarized intensity (colour) is overlaid with the $E$-vectors (red vectors). A reference of 100 per cent polarization is shown in the top right. (A colour version is available in the online article.) 

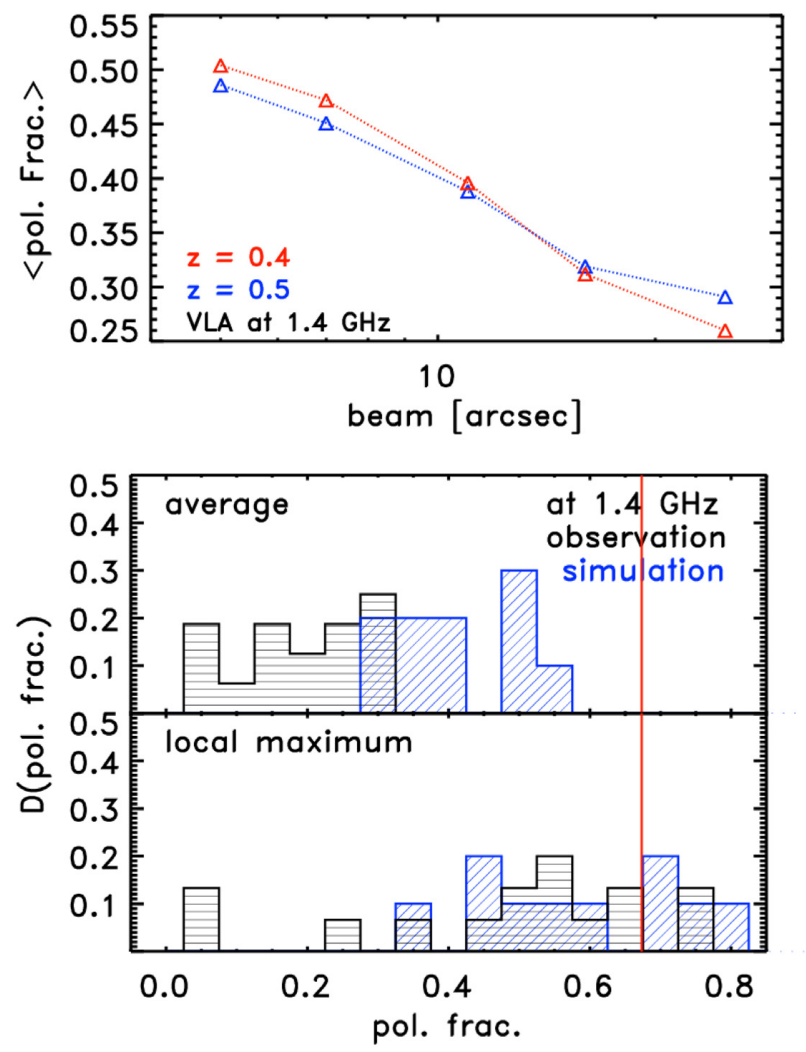

Figure 11. Top: radio-weighted degree of polarization as a function of the beam size. The two lines show the results for the VLA mock observations at redshift $z=0.4$ (red line) and $z=0.5$ (blue line). Bottom: distributions of the polarization fraction measured by observations (black) and in the simulation (blue) $1.4 \mathrm{GHz}$. The top distributions compare the average degree of polarization across the relic, while the bottom distributions compare the local maximum degree recorded in each relic. The red line marks the polarization fraction 75 per cent computed by Skillman et al. (2013). (A colour version is available in the online article.)

view, the relic's extent, along the LoS, is about $\sim 134 \mathrm{kpc}$ at the left edge and it increases to $\sim 434 \mathrm{kpc}$ at the right edge. In the side-on view, only the $E$-vectors that are close to the right edge (i.e. the shock front) align with each other. Finally, in the face-on view, their orientation is completely randomized by Faraday rotation due to the large RM of this projection. The position of the region coincides with the position of the regular and laminar pre-shock area where the magnetic field is aligned in front of the relic (see Fig. 6).

\subsubsection{Depolarized 'brush'}

In contrast to the strong alignment of $E$-vectors in region A, we observe that the $E$-vectors in region $\mathrm{C}$ are completely misaligned. This region looks similar to the 'brush' in 1RXS J0603.3+4214 (van Weeren et al. 2012). Moreover, in both the face-on and sideon view, the $E$-vectors in this region are randomly orientated. The random orientation in the face-on view can be explained by the large RM. But its nature in the edge-on and side-on views must be physical since their RM values are fairly low. We infer that the random orientations are due to the cold sub-clump falling on to the cluster in this region (see Fig. 6). The sub-clump stirs both the upstream gas and the upstream magnetic fields causing the magnetic field orientation to be random and the $E$-vectors not to align.
In summary, our simulation does not reproduce the morphology observed in relics such as CIZA J2242.8+5301 or Abell 2744, whereas, locally, we recover structures that have been found in observed radio relics. We conclude that threads and filaments can be very distant to each other along the LoS and hence they can probe magnetic field structures with different orientations. We found that aligned polarization vectors reside in the region of the shock where the upstream magnetic field is laminar, and that they are orientated randomly in the region with a disturbed upstream magnetic field.

\subsection{Magnetic field vectors and polarization direction}

It is possible to derive the magnetic field direction from the observed polarized emission (see equation 9), provided that one accounts for the effect of Faraday rotation. We derived the magnetic field direction for different frequencies between $0.15 \mathrm{GHz}$ and $4.85 \mathrm{GHz}$ using equation (9). We subtracted for the effect of Faraday rotation by subtracting $\langle\mathrm{RM}\rangle_{v_{\mathrm{obs}}} \lambda_{\mathrm{obs}}^{2}$ from each polarization angle. In our case, a good proxy for the orientation of the physical magnetic field of the relic is its radio-emission-weighted orientation, which we computed in the same frequency range (see Appendix B). We computed the differences, $\Delta \beta \in\left[-90^{\circ},+90^{\circ}\right]$, between the corrected polarization orientation, $B$-vectors and the radio-weighted orientation of the physical field. We also included the control case $(\mathrm{RM}=0)$ as this gives the intrinsic orientation of the polarized emission. Finally, we obtained a distribution of differences across the relic for each frequency and each LoS.

In Fig. 13, we plot the mean and the dispersion (i.e. using the standard deviation) of these distributions. Regardless of both frequency and LoS, the mean values fluctuate around $0^{\circ}$ and they never exceed $\pm 12^{\circ}$. Whenever we include Faraday rotation, the dispersion varies between $\sim 53^{\circ}$ at low frequencies and $17-35^{\circ}$ at high frequencies. The dispersion at low frequencies is significantly smaller, $<30^{\circ}$ for the control case (i.e. $\mathrm{RM}=0$ ). The edge-on and the face-on view cases including RM converge to the control case at high frequencies. On the other hand, there is no convergence to the control case in the edge-on view even at the highest frequencies that we tested. We conclude that radio observations can determine the intrinsic orientation of polarization if the amount of Faraday rotation along the LoS is small. Future observations and RM synthesis to control the foreground RM should make it possible to measure the local magnetic field. But we want to stress that this only holds provided that the RM along the LoS is sufficiently small. Furthermore, the dispersion of the intrinsic difference, i.e. if $\mathrm{RM}=$ 0 , is lower if the relic extent along the LoS is smaller.

\section{DISCUSSION AND CONCLUSION}

In this paper, we have studied the polarization of radio relics in cosmological simulations. This pilot study has the objective to explore the degree of realism of magnetic fields produced in these simulations and to highlight their current limitations. We combined the formalisms in Burn (1966) and Hoeft \& Brüggen (2007) to model the polarized radio emission of a radio relic. Our simulation grid gave us the possibility to study the relic seen in six different projections. For each projection, we included internal and external Faraday rotation as well as the control case without Faraday rotation. Furthermore, we computed the polarized emission for observing frequencies between $150 \mathrm{MHz}$ and $4.85 \mathrm{GHz}$ and we produced mock observations for the radio telescopes Effelsberg, VLA, and LOFAR. Our work aims at improving the present knowledge about the following key questions: 


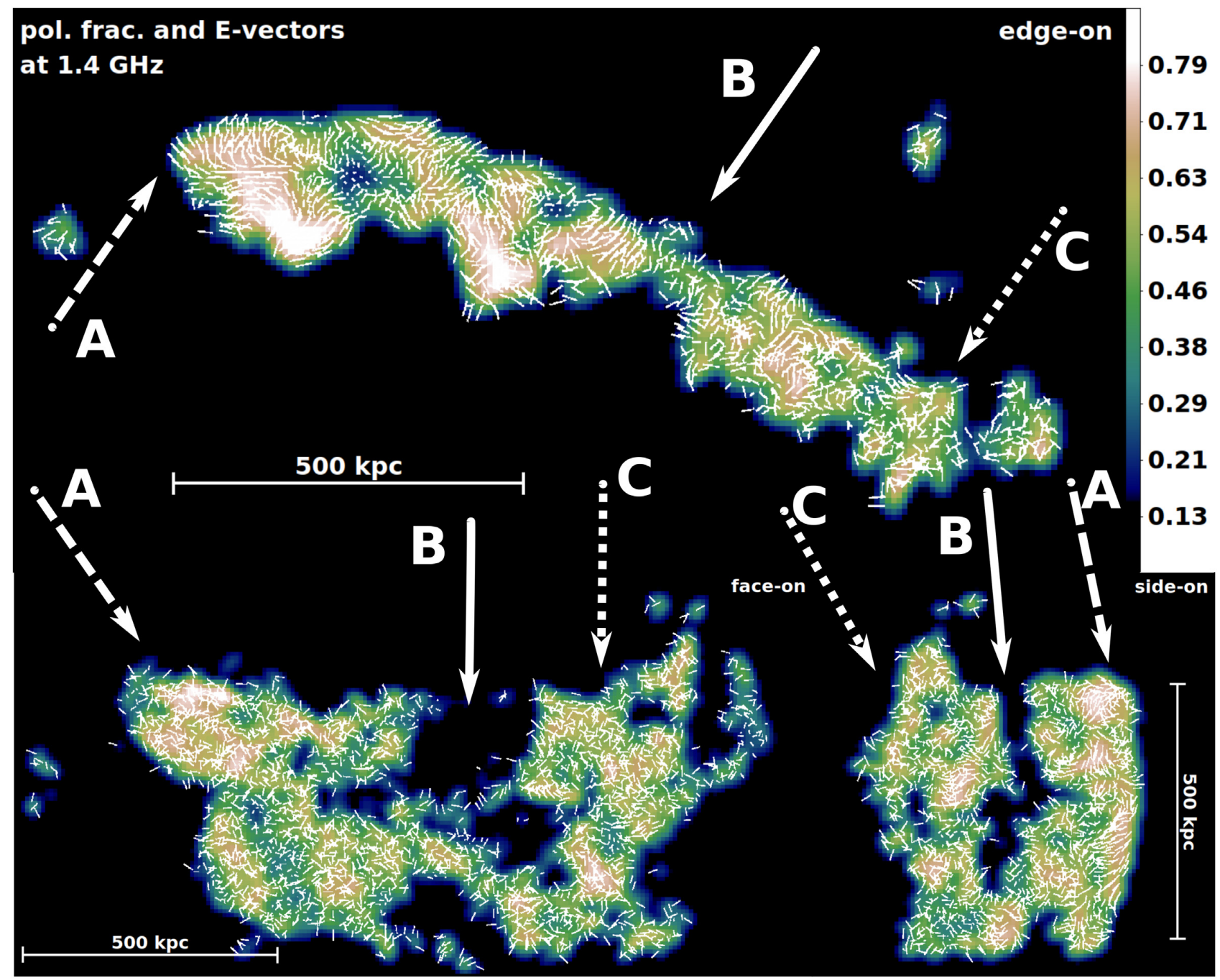

Figure 12. Polarization fraction (colour) overlaid with the corresponding $E$-vectors (white vectors). The three different panels show the relic seen in different projections. The letters highlight regions that have remarkable similarities with structures observed in real radio relics: (A) alignment of $E$-vectors, (B) a bridge and (C) a depolarized 'brush'. The vectors have been normalized to unity. (A colour version is available in the online article.)
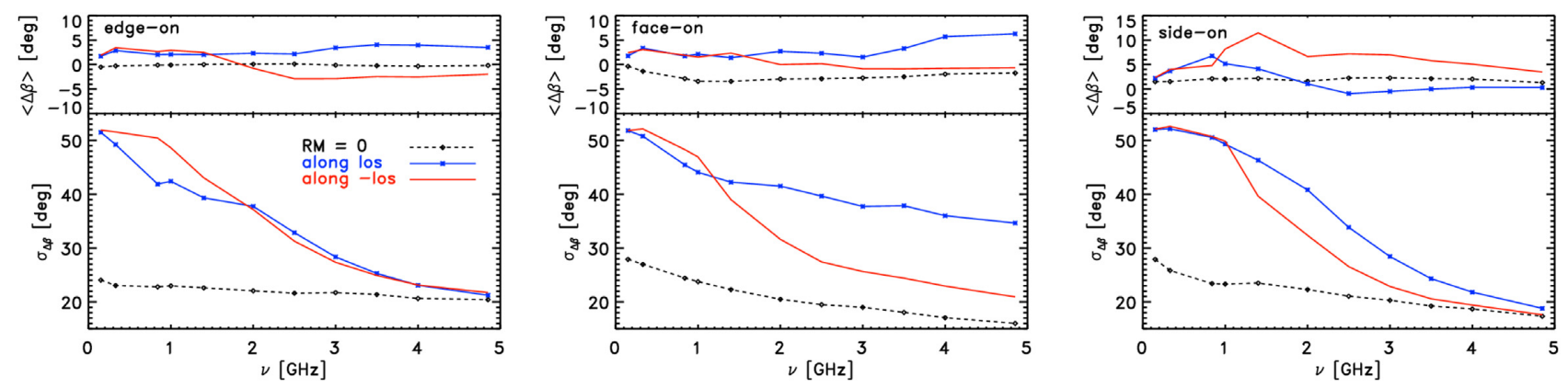

Figure 13. The plots show the average (top row) and corresponding standard deviation (bottom row) of the differences between the radio-emission-weighted magnetic field orientation and the magnetic field orientation derived from polarization. The black lines (dashed with diamonds) give the cases without RM, while the blue (solid with squares) and red (solid) lines give the cases with RM seen along the two different directions of the LoS. The different panels display the results for the three different projections. (A colour version is available in the online article.)

(i) What are the polarization properties of radio relics?

The average degree of polarization of the relic without Faraday rotation for frequencies above $1.0 \mathrm{GHz}$ is $\geq 50$ per cent and it slightly increases with frequency. These values are close to the intrinsic degree of polarization. Hence, the depolarization due to different magnetic field orientations along the LoS inside the 
emitting region is low. For frequencies below $1.0 \mathrm{GHz}$, the average degree of polarization decreases due to the growing size of the emitting region.

When including Faraday rotation, the average degree of polarization drops significantly for low observing frequencies. In this case, the strength of the depolarization depends on the position of the relic along the LoS and also the Faraday depth of the emitting structure, whereas for high frequencies, $\geq 1.0-2.0 \mathrm{GHz}$, the degree of polarization is closer to the cases without Faraday rotation. Therefore, we found a polarization degree significantly larger than that measured in observations. If the effect of beam depolarization is included, the degree of polarization decreases even at high frequencies (i.e. from $\sim 50$ per cent to $\sim 25$ per cent if the beam resolution goes from $5-25$ arcsec at $1.4 \mathrm{GHz}$ ).

(ii) What is the RM distribution in simulations?

We found asymmetric RM distributions with average values of a few 10-100 $\mathrm{rad} \mathrm{m}^{-2}$ and standard deviations, $\sigma_{\mathrm{RM}}$, that vary between a few tens $\mathrm{rad} \mathrm{m}^{-2}$ up to several hundred $\mathrm{rad} \mathrm{m}^{-2}$. These values agree with real RM measurements in galaxy clusters. Finally, we found that the shapes of the different RM distributions across our simulated relic are neither Gaussian nor symmetric, which is the usual assumption in several theoretical works (e.g. Burn 1966).

(iii) Can simulations reproduce the morphology of the observed polarized emission and does the morphology reflect the magnetic field structure in the relic region?

The simulated relic does not show the large-scale $(\geq 1-2 \mathrm{Mpc})$ polarization structures observed in real systems such as CIZA $\mathrm{J} 2242.8+5301$ or Abell 2744, where the $E$-vectors of the polarization tightly align with the shock normal. On the other hand, the simulation produces the observed structures on smaller scales $(\leq 200 \mathrm{kpc})$, i.e. the strong alignment of $E$-vectors as in CIZA $\mathrm{J} 2242.8+5301$, the depolarized 'brush' as in 1RXS J0603.3+4214 or a bridge. The latter is produced by a filamentary shock structure that would be visible if observed with sufficiently high resolution. These filaments are similar to the ones observed in 1RXS J0603.3+4214 and CIZA J2242.8+5301. Moreover, we found that the orientation of the $E$-vectors depends on the behaviour of the upstream magnetic field. All these similarities occur on scales that are comparable to the correlation length of the magnetic field (Vazza et al. 2018b; Domínguez-Fernández et al. 2019).

We report that the polarization $B$-vectors are reasonably aligned with the radio-emission-weighted direction of the magnetic field for $v \geq 3.0 \mathrm{GHz}$ if one corrects for the effect of Faraday rotation. Hence, despite a few cases in which a large column density along the LoS decorrelates the two vector fields, one can infer the magnetic field structure in the downstream relic region at these frequencies. We found that the magnetic field is neither only parallel nor entirely perpendicular to the shock normal, and therefore the polarized emission reflects the magnetic field and its correlation length limited to regions of a few hundred kpc.

Regardless of the LoS, we found very little to no correlation between magnetic field direction and polarization $B$-vectors for $v$ $<3.0 \mathrm{GHz}$. Additionally, there is an intrinsic misalignment, whose magnitude depends on the extent of the relic and the amount of residual emission along the LoS.

(iv) Can simulations reproduce the observed degree of polarization?

For frequencies above $>1 \mathrm{GHz}$, the average degree of polarization across the simulated relic is more than $>50$ per cent and, thus, it is significantly larger than in observations. However, the simulated relic shows local peaks of $\sim 70$ per cent, which is in line with local measurements in observation. Our findings agree with the results of
Skillman et al. (2013) (the only other numerical simulation using MHD in cosmology that we can compare to) who also measured a maximum degree of polarization of $\approx 75$ per cent.

A few radio-bright cells in our simulation determine the degree of polarization and hence it mirrors the intrinsic properties of these cells. Increasing the numerical resolution would produce a magnetic field that is more tangled on smaller scales. As a consequence, the average degree of polarization could decrease even if the individual cells have a high degree of polarization.

The average degree of polarization is reduced to 4-44 per cent in the mock observations of our relic, depending on redshift, beam size and observing frequency. This is in reasonable agreement with current radio observations. However, the Effelsberg mock observations do not reproduce the high degree of polarization measured by Kierdorf et al. (2017) for the relics in CIZA J2242.8+5301 and 1RXS J0603.3+4214. The fact that our results show discrepancies with these observations is due to either a correlation length in our simulation that is too small or due to missing micro-physics at the shock front.

(v) Which simulated relic properties are most different from observations?

The average Mach number of $M>2$ shocks that produces the relic is $\langle M\rangle \sim 2.4$, while the Mach number derived using the spectral index is $\sim 3.5$. Therefore, they are of the same order as estimates from observations (e.g. Hoang et al. 2018). Our relic is $\sim 1.7 \mathrm{Mpc}$ away from the cluster centre, which is similar to the observed relics (see Table 1). Yet, we noticed that when seen edge-on, its largest linear size is smaller than most of the observed relics. This projection's extent along the LoS is larger than the estimations for the relic in CIZA J2242.8+5301 (i.e. $250 \mathrm{kpc}$ from Kierdorf et al. 2017). Therefore, it is difficult to generalize our findings to the different geometries found in real systems. We require more simulated radio relics in order to better understand the observations.

At the relic position, we found an average magnetic field of $\sim 0.2$ $0.4 \mu \mathrm{G}$ and a radio-weighted magnetic field of $\sim 0.5 \mu \mathrm{G}$. These values are consistent with the lower limits estimated in most of the observations (e.g. see fig. 14 in Bonafede et al. 2013). It is just in the case of Abell 3667 (Finoguenov et al. 2010) where we find that the magnetic field strength obtained in the simulation is in discrepancy with observations as they report a strength of $\sim 3 \mu \mathrm{G}$ in the relic region.

The RM and its dispersion, $\sigma_{\mathrm{RM}}$, are of the same order as the observed RM (e.g. Bonafede et al. 2013; Böhringer et al. 2016). Our work supports that these values vary with respect to the cluster centre depending on two factors: the impact parameter and the specific LoS. In the most extreme cases, the variation can be of a factor of $\sim 10$. Finally, we can say that our simulation produces realistic magnetic field strengths, but in order to reproduce the same $\sigma_{\mathrm{RM}}$ with a higher magnetic field strength (as in the case of Abell 3667), smaller correlation lengths are required.

Our results show that the polarized emission of radio relics should strongly depend on the environment and the orientation of the polarization changes with the properties of the upstream gas. Laminar gas flows in the upstream produce a parallel alignment of the $E$-vectors, while disturbances in the upstream will cause a random orientation. This might reflect the local correlation length of the magnetic field. We also found that high-resolution observations above $2.0-3.0 \mathrm{GHz}$ will be able to reasonably estimate the magnetic field direction in the relic regions, provided that one corrects for Faraday rotation. In general, this can be done using high-resolution Faraday spectra. 
The fluctuation of the magnetic field on small scales points towards a small-scale field that is aligned by the shock on microscopic scales to explain the observed degrees of polarization. We are aware that adding micro-physics to our simulations will help us to understand better the observed degree of polarization in relics. Nevertheless, this will remain a task for future work.

It is not possible to make any conclusive assessment on the large-scale alignment of polarized emission observed in many radio relics (e.g. CIZA J2242.8+5301, 1RXS J0603.3+4214 etc) due to our small statistical sample. Either they are produced by ordered large-scale magnetic fields, or they are the result of compressed tangled magnetic fields. The two options are found on scales of $\leq 200 \mathrm{kpc}$ in different regions of the same simulated relic. Therefore, more simulated relics (also including strong shocks) are required to generate meaningful statistics.

In conclusion, some of our results may quantitatively change if we use a higher spatial resolution, which is difficult to reach at the moment. In particular, some of the small-scale details in our relic have the same size as the effective resolution of the Dedner cleaning applied in our MHD scheme and therefore magnetic structures on scales $\leq 32 \mathrm{kpc}$ may be affected by numerical diffusion. Hence, they would show fewer structures in reality, provided that the magnetic Prandtl number is $P_{\mathrm{m}} \leq 1$ and the magnetic Reynolds number is $R_{\mathrm{m}} \gg 10^{2}$ in the real ICM. Lastly, the numerical resolution might also affect the correlation length of the magnetic field (e.g. Donnert et al. 2018)

Our study used a model of relativistic electrons that is still relatively crude and that can be improved. First, our model assumes a constant magnetic field and downstream velocity for computing the ageing of the radio-emitting electrons in the downstream region. Secondly, our simulation does not include the injection of relativistic particles and magnetic fields from active galactic nuclei and radio galaxies. In principle, this might affect the shape and size of radio relics as argued by Nuza et al. (2017). Further uncertainties are related to the assumed acceleration efficiencies and the magnetic field. In this work, we assumed acceleration efficiencies that solely depend on the Mach number, while recent studies (e.g. Caprioli \& Spitkovsky 2014a; Wittor et al. 2017; Kang \& Ryu 2018) have shown that they can depend on additional parameters such as the shock obliquity. Finally, it is unclear if different magnetic field seeding mechanisms change the magnetic field morphology in the relic's environment. The goal of future studies would be to improve this modelling by including tailored AMR schemes dedicated to increasing the resolution at the relic and also to increase the statistics of simulated relics.

\section{ACKNOWLEDGEMENTS}

We thank our anonymous referee for the helpful comments, which helped us to improve the quality of our work. The cosmological simulations described in this work were performed using the ENZO code (http://enzo-project.org), which is the product of a collaborative effort of scientists at many universities and national laboratories. We gratefully acknowledge the ENZO development group for providing extremely helpful and well maintained online documentation and tutorials.

The authors gratefully acknowledge the Gauss Centre for Supercomputing e.V. (www.gauss-centre.eu) for supporting this project by providing computing time through the John von Neumann Institute for Computing (NIC) on the GCS Supercomputer JUWELS at Jülich Supercomputing Centre (JSC), under project nos. 11823 , 10755 and 9016 as well as hhh42, hhh44 and stressicm.
The original simulations on which this work is based were produced under project HHH42 at JSC by FV as PI. DW, FV and PDF acknowledge financial support from the European Union's Horizon 2020 program under the ERC Starting Grant 'MAGCOW', no. 714196. We also acknowledge the usage of online storage tools kindly provided by the Inaf Astronomica Archive (IA2) initiave (http://www.ia2.inaf.it).

$\mathrm{MH}$ acknowledges support by the BMBF Verbundforschung under the grant $05 \mathrm{~A} 17 \mathrm{STA}$

We acknowledge fruitful scientific discussion with A. Bonafede, K. Rajpurohit, C. Stuardi, G. Brunetti and S. O'Sullivan. This research made use of the radio astronomical database galaxyclusters.com, maintained by the Observatory of Hamburg.

\section{REFERENCES}

Ackermann M. et al., 2014, ApJ, 787, 18

Akahori T., Ryu D., 2010, ApJ, 723, 476

Akahori T., Ryu D., 2011, ApJ, 738, 134

Akamatsu H. et al., 2017, A\&A, 600, A100

Arshakian T. G., Beck R., 2011, MNRAS, 418, 2336

Berger M. J., Colella P., 1989, J. Comput. Phys., 82, 64

Blandford R. D., Ostriker J. P., 1978, ApJ, 221, L29

Böhringer H., Chon G., Kronberg P. P., 2016, A\&A, 596, A22

Bonafede A. et al., 2009a, A\&A, 503, 707

Bonafede A., Giovannini G., Feretti L., Govoni F., Murgia M., 2009b, A\&A, 494, 429

Bonafede A. et al., 2012, MNRAS, 426, 40

Bonafede A., Vazza F., Brüggen M., Murgia M., Govoni F., Feretti L. Giovannini G., Ogrean G., 2013, MNRAS, 433, 3208

Bonafede A., et al., 2015, Advancing Astrophysics with the Square Kilometre Array (AASKA14), Sissa Medialab, Giardini Naxos, Italy, p. 95

Botteon A., Gastaldello F., Brunetti G., Kale R., 2016, MNRAS, 463, 1534

Botteon A., Brunetti G., Ryu D., Roh S., 2019, preprint (arXiv:1907:00966)

Brentjens M. A., de Bruyn A. G., 2005, A\&A, 441, 1217

Brunetti G., Jones T. W., 2014, Int. J. Modern Phys. D, 23, 1430007

Bryan G. L. et al., 2014, ApJS, 211, 19

Burn B. J., 1966, MNRAS, 133, 67

Caprioli D., Spitkovsky A., 2014a, ApJ, 783, 91

Caprioli D., Spitkovsky A., 2014b, ApJ, 794, 46

Clarke T. E., Ensslin T. A., 2006, AJ, 131, 2900

Colella P., Glaz H. M., 1985, J. Comput. Phys., 59, 264

de Gasperin F., van Weeren R. J., Brüggen M., Vazza F., Bonafede A., Intema H. T., 2014, MNRAS, 444, 3130

de Gasperin F., Intema H. T., van Weeren R. J., Dawson W. A., Golovich N., Wittman D., Bonafede A., Brüggen M., 2015, MNRAS, 453, 3483

Dedner A., Kemm F., Kröner D., Munz C.-D., Schnitzer T., Wesenberg M., 2002, J. Comput. Phys., 175, 645

Di Gennaro G. et al., 2018, ApJ, 865, 24

Domínguez-Fernández P., Vazza F., Brüggen M., Brunetti G., 2019, MNRAS, 486, 623

Donnert J., Vazza F., Brüggen M., ZuHone J., 2018, Space Sci. Rev., 214, 122

Ensslin T. A., Biermann P. L., Klein U., Kohle S., 1998, A\&A, 332, 395

Feretti L., Bacchi M., Slee O. B., Giovannini G., Govoni F., Andernach H., Tsarevsky G., 2006, MNRAS, 368, 544

Ferrari C., Govoni F., Schindler S., Bykov A. M., Rephaeli Y., 2008, Space Sci. Rev., 134, 93

Finoguenov A., Sarazin C. L., Nakazawa K., Wik D. R., Clarke T. E., 2010, ApJ, 715, 1143

Golovich N., van Weeren R. J., Dawson W. A., Jee M. J., Wittman D., 2017, ApJ, 838, 110

Golovich N. et al., 2019, ApJS, 882, 69,

Golovich N. et al., 2019, ApJS, 240, 39 
Govoni F., Murgia M., Xu H., Li H., Norman M. L., Feretti L., Giovannini G., Vacca V., 2013, A\&A, 554, A102

Guo X., Sironi L., Narayan R., 2014, ApJ, 797, 47

Ha J.-H., Ryu D., Kang H., 2018, ApJ, 857, 26

Hamaker J. P., Bregman J. D., Sault R. J., 1996, A\&AS, 117, 137

Hammond A. M., Robishaw T., Gaensler B. M., 2012, preprint (arXiv: 1209.1438)

Hoang D. N. et al., 2018, MNRAS, 478, 2218

Hockney R. W., Eastwood J. W., 1988, Computer Simulation using Particles, Taylor \& Francis Group, New York

Hoeft M., Brüggen M., 2007, MNRAS, 375, 77

Hong S. E., Kang H., Ryu D., 2015, ApJ, 812, 49

Iapichino L., Brüggen M., 2012, MNRAS, 423, 2781

Johnston-Hollitt M., 2004, in Reiprich T., Kempner J., Soker N., eds, The Riddle of Cooling Flows in Galaxies and Clusters of Galaxies, Univ. Virgina, Charlottesville, VA, USA, p. 15

Johnston-Hollitt M. et al., 2015, Advancing Astrophysics with the Square Kilometre Array (AASKA14), Sissa Medialab, Giardini Naxos, Italy, p. 92

Kale R., Dwarakanath K. S., Bagchi J., Paul S., 2012, MNRAS, 426, 1204

Kang H., Ryu D., 2018, ApJ, 856, 33

Kierdorf M., Beck R., Hoeft M., Klein U., van Weeren R. J., Forman W. R., Jones C., 2017, A\&A, 600, A18

Klein U., Fletcher A., 2015, Galactic and Intergalactic Magnetic Fields, Springer International Publishing, Heidelberg, Germany

Laing R. A., 1980, MNRAS, 193, 439

Lindner R. R. et al., 2014, ApJ, 786, 49

Loi F. et al., 2017, MNRAS, 472, 3605

Markevitch M., Govoni F., Brunetti G., Jerius D., 2005, ApJ, 627, 733

Nuza S. E., Gelszinnis J., Hoeft M., Yepes G., 2017, MNRAS, 470, 240

O'Sullivan S. P. et al., 2019, A\&A, 622, A16

Otmianowska-Mazur K., Soida M., Kulesza-Żydzik B. Hanasz M., Kowal G., 2009, ApJ, 693, 1

Pearce C. J. J. et al., 2017, ApJ, 845, 81

Pinzke A., Oh S. P., Pfrommer C., 2013, MNRAS, 435, 1061

Rajpurohit K. et al., 2018, ApJ, 852, 65

Rudnick L., Brown S., 2009, AJ, 137, 145

Rybicki G. B., Lightman A. P., 1986, Radiative Processes in Astrophysics, Wiley-VCH, Weinheim, Germany, p. 400

Sault R. J., Hamaker J. P., Bregman J. D., 1996, A\&AS, 117, 149

Shimwell T. W., Markevitch M., Brown S., Feretti L., Gaensler B. M., Johnston-Hollitt M., Lage C., Srinivasan R., 2015, MNRAS, 449, 1486

Shimwell T. W. et al., 2017, A\&A, 598, A104

Skillman S. W., Xu H., Hallman E. J., O'Shea B. W., Burns J. O., Li H., Collins D. C., Norman M. L., 2013, ApJ, 765, 21

Sokoloff D. D., Bykov A. A., Shukurov A., Berkhuijsen E. M., Beck R., Poezd A. D., 1998, MNRAS, 299, 189

Tribble P. C., 1991, MNRAS, 250, 726

van Weeren R. J., Röttgering H. J. A., Brüggen M., Hoeft M., 2010, Science, 330, 347

van Weeren R. J., Brüggen M., Röttgering H. J. A., Hoeft M., Nuza S. E., Intema H. T., 2011a, A\&A, 533, A35

van Weeren R. J., Hoeft M., Röttgering H. J. A., Brüggen M., Intema H. T., van Velzen S., 2011b, A\&A, 528, A38

van Weeren R. J., Röttgering H. J. A., Intema H. T., Rudnick L., Brüggen M., Hoeft M., Oonk J. B. R., 2012, A\&A, 546, A124

van Weeren R. J. et al., 2013, ApJ, 769, 101

van Weeren R. J. et al., 2017, ApJ, 835, 197

van Weeren R. J., de Gasperin F., Akamatsu H., Brüggen M., Feretti L., Kang H., Stroe A., Zandanel F., 2019, Space Sci. Rev., 215, 16

Vazza F., Brüggen M., 2014, MNRAS, 437, 2291

Vazza F., Brunetti G., Gheller C., 2009, MNRAS, 395, 1333

Vazza F., Eckert D., Brüggen M., Huber B., 2015, MNRAS, 451, 2198

Vazza F., Brüggen M., Hinz P. M., Wittor D., Locatelli N., Gheller C., 2018a, MNRAS, 480, 3907
Vazza F., Brunetti G., Brüggen M., Bonafede A., 2018b, MNRAS, 474, 1672

Wittor D., Vazza F., Brüggen M., 2017, MNRAS, 464, 4448

\section{APPENDIX A: SYNCHROTRON EMISSION}

In this section, we summarize the mathematical details used to compute the downstream profile of the radio emission (see Section 2.2). Following the approach of Hoeft \& Brüggen (2007), we compute the emission per volume and its parallel and perpendicular components at a distance $x$ away from the shock front as the convolution of the electron spectrum $n_{\mathrm{E}}(\tau, x)$ and the function $F\left(1 / \tau^{2}\right)$ :

$$
\begin{aligned}
& \frac{\mathrm{d} P}{\mathrm{~d} V \mathrm{~d} \nu}(x)=C_{\mathrm{R}} \int_{0}^{E_{\max }} n_{\mathrm{E}}(\tau, x) F\left(\frac{1}{\tau^{2}}\right) \mathrm{d} \tau \\
& \frac{\mathrm{d} P_{\perp}}{\mathrm{d} V \mathrm{~d} \nu}(x)=C_{\mathrm{R}} \int_{0}^{E_{\max }} n_{\mathrm{E}}(\tau, x)\left[F\left(\frac{1}{\tau^{2}}\right)-G\left(\frac{1}{\tau^{2}}\right)\right] \mathrm{d} \tau \\
& \frac{\mathrm{d} P_{\|}}{\mathrm{d} V \mathrm{~d} \nu}(x)=C_{\mathrm{R}} \int_{0}^{E_{\max }} n_{\mathrm{E}}(\tau, x)\left[F\left(\frac{1}{\tau^{2}}\right)+G\left(\frac{1}{\tau^{2}}\right)\right] \mathrm{d} \tau .
\end{aligned}
$$

The functions $F(x)$ and $G(x)$ depend on the modified Bessel functions $K$ as (see Rybicki \& Lightman 1986):

$$
\begin{aligned}
& F(x)=x \int_{x}^{\infty} K_{\frac{5}{3}}(\xi) \mathrm{d} \xi \\
& G(x)=x K_{\frac{2}{3}}(x) .
\end{aligned}
$$

The constant $C_{\mathrm{R}}$ is computed as:

$$
C_{\mathrm{R}}=\frac{9 e^{5 / 2} B^{3 / 2} \sin \alpha}{4 \sqrt{\nu_{\mathrm{obs}} m_{\mathrm{e}} c}},
$$

where $m_{\mathrm{e}}$ and $e$ are the electron mass and charge, $B$ is the magnetic field, $\alpha$ is the pitch angle, $v_{\text {obs }}$ is the observing frequency, $c$ is the speed of light and $E$ is the electron energy. The function $\tau$ depends on the energy as:

$\tau=\sqrt{\frac{3 e B}{16 v_{\mathrm{obs}} m_{\mathrm{e}} c}}\left(\frac{E}{m_{\mathrm{e}} c^{2}}+1\right)$.

The electron spectrum at a distance $x$ to the shock is computed as:

$$
\begin{aligned}
n_{\mathrm{E}}(E, x)= & \frac{n_{\mathrm{e}} C_{\text {spec }}}{m_{\mathrm{e}} c^{2}}\left(\frac{E}{m_{\mathrm{e}} c^{2}}\right)^{-s} \\
& \times\left[1-\left(\frac{m_{\mathrm{e}} c^{2}}{E_{\max }}+C_{\mathrm{cool}} \frac{x}{v_{\mathrm{d}}}\right) \frac{E}{m_{\mathrm{e}} c^{2}}\right]^{s-2} .
\end{aligned}
$$

Electrons can only be accelerated to a finite energy $E_{\max }$. Hence, the spectrum is evaluated if $E C_{\text {cool }} x / v_{\mathrm{d}} / m_{\mathrm{e}} / c^{2}<1-E / E_{\max }$, with $v_{\mathrm{d}}$ being the downstream velocity of the shock. The cooling constant is given as:

$C_{\mathrm{cool}}=\frac{\sigma_{\mathrm{T}}}{6 m_{\mathrm{e}} c \pi}\left(B_{\mathrm{CMB}}^{2}+B^{2}\right)$.

Here, $\sigma_{\mathrm{T}}$ is the Thomson cross-section and $B_{\mathrm{CMB}}^{2}$ is the equivalent magnetic field of the cosmic microwave background at redshift $z$. The normalization of the spectrum is:

$C_{\text {spec }}=\xi_{\mathrm{e}} \frac{u_{\mathrm{d}}}{c^{2}} \frac{m_{\mathrm{p}}}{m_{\mathrm{e}}} \frac{q-1}{q} \frac{1}{I_{\mathrm{spec}}}$,

where $\xi_{\mathrm{e}}$ is the acceleration efficiency, $q$ is the entropy jump across the shock, $u_{\mathrm{d}}$ is the internal energy of the downstream gas and $m_{\mathrm{p}}$ is the proton mass. The integral $I_{\text {spec }}$ is given as

$I_{\mathrm{spec}}=\int_{E_{\min }}^{\infty} E\left(\frac{E}{m_{\mathrm{e}} c^{2}}\right)^{-s}\left(1-\frac{E}{E_{\max }}\right)^{s-2} \mathrm{~d} E$. 
For the minimum energy, above which electrons are considered to be suprathermal, we chose $E_{\min }=10 k_{\mathrm{b}} T$.

\section{APPENDIX B:AVERAGE OF ANGLES}

We use equation (5) to compute the radio-weighted average of a physical quantity $Q$. If $Q$ is an angle $\alpha$, we first compute the two components: $x=\sin (\alpha)$ and $y=\cos (\alpha)$. Next, we compute the radio-weighted averages of the two components $\langle x\rangle_{\nu_{\mathrm{obs}}}$ and $\langle y\rangle_{v_{\mathrm{obs}}}$. Finally, we compute the radio-weighted average angle as:

$\langle\alpha\rangle_{v_{\mathrm{obs}}}=\arctan \left(\frac{\langle x\rangle_{v_{\mathrm{obs}}}}{\langle y\rangle_{v_{\mathrm{obs}}}}\right)$.

This approach is reasonable if the scatter of angles is about $90^{\circ}$ or less. In our case, this works well for almost all LoS, since a few cells dominate the radio emission. We applied this algorithm when we computed the radio-weighted orientation of the physical magnetic field (see Section 3.7). This is not to be confused with the orientation of the polarization vectors, which we computed using equations (6) and (9).

\section{APPENDIX C: THE CORRELATION OF THE MAGNETIC SPECTRUM}

The magnetic power spectrum in a magnetized plasma has a characteristic shape that differs from the shape of the kinetic power spectrum. In detail, it cannot be characterized only by a power law. The galaxy cluster produced by our MHD simulation has enough resolution for better resolving the morphology of magnetic fields at small scales during structure formation. Therefore, we can better constrain the shape of the magnetic power spectrum. The magnetic spectral properties of the cluster have already been analysed in Domínguez-Fernández et al. (2019). One important result of this work is that, despite the different dynamical states, the magnetic spectra can be well fitted by the following equation:

$E_{M}(k)=A k^{3 / 2}\left[1-\operatorname{erf}\left[B \ln \left(\frac{k}{C}\right)\right]\right]$

where $A$ is the normalization, $B$ is related to the width of the spectra and $C$ is a characteristic wavenumber that inverse corresponds to the inverse outer scale of the magnetic field. In this work, we computed the power spectrum of our data and then fitted it to equation $(\mathrm{C} 1)$. In this work, we refer to the correlation length given by the outer scale of the magnetic spectrum, i.e. the $C$ parameter.

This paper has been typeset from a $\mathrm{T}_{\mathrm{E}} \mathrm{X} / \mathrm{LT} \mathrm{E} \mathrm{X}$ file prepared by the author. 